This is a postprint version of the following published document:

Valverde-Albacete, F. J. \& Peláez-Moreno, C. (2015). The spectra of irreducible matrices over completed idempotent semifields. Fuzzy Sets and Systems, 271, 46-69.

DOI: http://dx.doi.org/10.1016/j.fss.2014.09.022

(C) 2014 Elsevier B.V.

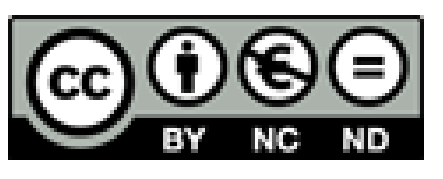

This work is licensed under a Creative Commons Attribution-NonCommercialNoDerivatives 4.0 International License. 


\title{
The spectra of irreducible matrices over completed idempotent semifields
}

\author{
Francisco J. Valverde-Albacete ${ }^{a}$, Carmen Peláez-Moreno ${ }^{\mathrm{b}, *}$ \\ a Departamento de Lenguajes y Sistemas Informáticos, Universidad Nacional de Educación a Distancia, 28040 Madrid, Spain \\ b Departamento de Teoría de la Señal y de las Comunicaciones, Universidad Carlos III de Madrid, 28911 Leganés, Spain \\ *E-mail addresses: fva@1si.uned.es (F.J. Valverde-Albacete), carmen@tsc.uc3m.es (C. Peláez-Moreno).
}

\begin{abstract}
Motivated by some spectral results in the characterization of concept lattices we investigate the spectra of reducible matrices over complete idempotent semifields in the framework of naturally-ordered semirings, or dioids. We find non-null eigenvectors for every non-null element in the semifield and conclude that the notion of spectrum has to be refined to encompass that of the incomplete semifield case so as to include only those eigenvalues with eigenvectors that have finite coordinates. Considering special sets of eigenvectors brings out finite complete lattices in the picture and we contend that such structure may be more important than standard eigenspaces for matrices over completed idempotent semifields.
\end{abstract}

Keywords: Matrix spectra; Dioids; Complete idempotent semifields; Complete idempotent semimodules; Spectral order lattices

\section{Introduction}

Several attempts have been made to generalise the basic framework of Formal Concept Analysis (FCA) [1] or Galois lattice theory [2] since it was conceived. Recall that this is a theory of concrete lattices arising from certain Galois connections between two sets induced by a binary incidence relation. It finds concrete applications in data mining and exploratory information retrieval, among others [3].

Perhaps the earliest and more developed generalisation is that of Formal Concept Analysis in a Fuzzy Setting, where incidences are allowed to have values in a fuzzy algebra which is also a complete lattice $[4,5]$. Such fuzzy algebras can alternatively be described as fuzzy semirings [6]. Recall that a semiring is an algebra $\mathcal{S}=\langle S, \oplus, \otimes$, $\epsilon, e\rangle$ whose additive structure, $\langle S, \oplus, \epsilon\rangle$, is a commutative monoid and whose multiplicative structure, $\langle S \backslash\{\epsilon\}, \otimes$, $e\rangle$, is a monoid with multiplication distributing over addition from right and left and with additive neutral element absorbing for $\otimes$, i.e. $\forall a \in S, \epsilon \otimes a=\epsilon[6]$. 
An independently motivated generalisation of FCA, $\mathcal{K}$-Formal Concept Analysis, uses an idempotent semi-field $\mathcal{K}-$ a kind of semiring with a multiplicative group structure-as the range of the relation [7]. Whereas fuzzy semirings are mostly used to capture a "degree of truth", semifields are used to capture the concept of "cost" or "utility".

It is intriguing that these algebras induce Galois connections and Formal Concept Analysis inasmuch as idempotent semifields are as far as a naturally ordered semiring can be from prototypical fuzzy semirings like $\langle[0,1]$, max, min, 0,1$\rangle$-in a sense made evident in this paper. In fact, idempotent semifields do not fulfil some of the more restrictive or technical conditions for an algebra $L$ to define an $L$-fuzzy set [8]: in particular, in an idempo-tent semifield the identity is never an infinity element.

However, it has already been determined that the condition for an algebra to induce a flavour of FCA is that it be a complete residuated lattice [5]. Unsurprisingly, one of the notoriously overlooked abstractions of fuzzy semirings and idempotent semifields are dioids, or naturally-ordered semirings whose zero is the bottom in the order. Dioids are already residuated so complete dioids are already complete residuated lattices (see Fig. 1), hence Formal Concept Analysis-inducing. Furthermore, semiring 2 is embedded in both fuzzy semirings and idempotent semifields. Note that Ref. [9] already asked in this venue for a revisiting of idempotent semifields and the investigation of their relationship to fuzzy algebras.

On the other hand, concept lattices as issued from standard FCA show a remarkable relation to some eigenspaces of the incidence relation. For instance Ref. [10] found that the formal concepts in $\mathcal{K}$-Formal Concept Analysis could be defined by means of the eigenequation of the unit eigenvalue. Building on earlier work, Ref. [11] demonstrated that binary formal concepts were optimal factors for decomposing a Boolean matrix, while Ref. [12] extended this to formal concepts over a residuated lattice. Both kind of results hint strongly that Formal Concept Analysis has some relationship with the Singular Value Decomposition (SVD) of the incidence relation and that formal concepts are pairs of left/right singular vectors. Despite the spectral theory of dioids having a long history of results [6], few general results for the cases of interest are known [13,14] and a theorem of spectral decomposition is undiscovered, to the best of our knowledge.

This work tries to pave the way for an overarching theory of Formal Concept Analysis over complete dioids by trying to make explicit the relation from the other side of the picture: between complete lattices and some eigenspaces related to relations with entries in a complete (residuated) dioid. Unfortunately, idempotent semifields, except 2 are all incomplete, what seems to doom our efforts in this direction. However, it is well-known that idempotent semifields can easily be completed: the problem with the bottom being its lack of inverse, we can easily prescribe the top $T$ to take this role.

This paper is dedicated to exploring the consequences of this decision in what respects the spectral theory of matrices over such top-completed idempotent semifields. We will prove that, far from harming our initial intentions, completing the semifield unveils lattice structures as scaffoldings of eigenspaces, and that such structures extend to more general semirings.

Also, we point out noticeable differences with the spectrum of incomplete idempotent semifields. To start with, since $T$ may be a coordinate in eigenvectors, the spectrum is more extensive, to the point where, once a non-null eigenvalue is found, most of the values in the dioid are spectral, albeit their eigenvectors will have non-finite coordinates (Section 3.1). This necessitates the definition of the proper right (left) spectrum, $\mathrm{P}^{\mathrm{P}}(A)$ whose corresponding eigenvectors have some finite coordinate, which partially recovers the picture in the incomplete, irreducible case (Section 3.2).

Furthermore, once $\overline{\mathcal{K}}$ is a completed idempotent semifield, $\top$ may be an eigenvalue (Section 3.1.3), whence the structure of the eigenspaces is that of a complete lattice. Therefore, independency of eigenvectors plays a lesser role than heretofore expected. Rather, in our analysis, the order properties of such eigenvectors-induced from the order in the underlying semiring-are highlighted (Section 3.2.1).

This paper is organized as follows: Section 2 delimits the area of application of our findings by presenting a family picture of semirings (Section 2.1) followed by a discussion of completeness issues in idempotent semifields (Section 2.2). Then we state formally the eigenproblem on semirings as well as some techniques to solve it in dioids (Section 2.3). A review of the different cryptomorphisms or interpretations of matrices over semirings as number arrays, relations or networks with weights in a semiring, crucial for the spectral theory, can also be found in Section 2.4. Section 3 presents our results for the spectra of irreducible matrices over completed idempotent semifields beginning with a compilation and contextualization of previous results about the null eigenvalue and eigenspace (Section 3.1) 
also useful for the reducible case, tackled in [15]. The spectra are finally characterized in Section 3.2 including a discussion about the role of this eigenvectors for the representation of eigenspaces (Section 3.2.1). Then, we illustrate our findings with examples (Section 4), discuss the existent solutions for the incomplete case (Section 5), and draw some conclusions in Section 6.

\section{Preliminaries}

\subsection{Semirings: a family picture}

Considering the enrichment of the properties of semirings, it is a well known result that the multiplicative and additive structures are completely independent, what accounts for their abundance [6,14]. They also have different importance in the classification and usability of semirings, as shown in Fig. 1, a "family picture" of commutative semirings as a concept lattice [1].

Focusing on the additive structure, a semiring is (additively) cancellative if for $a, b, c \in S$ when $a \oplus b=a \oplus c$ implies $b=c$. Of course, a ring is a cancellative semiring whose additive structure is that of a group, $\forall a \in S, \exists c \in$ $S, a \oplus c=\epsilon$. On the other hand, a semiring is zerosumfree if for $a, b \in S$ when $a \oplus b=\epsilon$ then $a=b=\epsilon$. Compared to a ring, zerosumfree semirings crucially lack additive inverses. In fact, rings are as "far away" as possible from zerosumfree semirings, the singleton $\mathbb{1}=\{\epsilon\}$ being the only semiring that is both. See for instance the locations of $\mathbb{N}_{0}, \mathbb{B}$ and $\mathbb{Z}$ in Fig. 1.

A semiring $\mathcal{S}$ is partially-ordered iffthere exists a partial order relation $\langle S, \leq\rangle$ compatible with addition and multiplication, such that for all $a, b, c \in S$, if $a \leq b$ then $a \oplus c \leq b \oplus c, a \otimes c \leq b \otimes c$ and $c \otimes a \leq c \otimes b$. In partially-ordered semirings, if $a_{i} \leq b_{i}$ then $\sum a_{i} \leq \sum b_{i}$. Furthermore, if $\mathcal{S}$ is a partially-ordered set, then it is positive [16] if $\epsilon=\perp$ is the infimum or bottom for this set $\perp \leq a$, for all $a \in S$. If $\mathcal{S}$ is positive then it is zerosumfree and also if $a_{i} \leq b_{i}$ then $\prod a_{i} \leq \prod b_{i}[16]$.

In a semiring, the natural or canonical or difference pre-order is for all $a, b \in S, a \preccurlyeq b \Longleftrightarrow a \oplus c=b$ for some $c \in S$. A semiring $\mathcal{D}=\langle D, \oplus, \otimes, \perp, e\rangle$ is a dioid-for double monoid-or naturally-or canonically- [14] or difference-ordered [6,16,17] if $a \preccurlyeq b$ and $b \preccurlyeq a$ entails $a=b$ for $a, b \in D$. This is clearly a partial-order [14, Chap. 1, Prop. 6.1.7] with $\perp=\epsilon$, hence dioids are positive semirings, whence also zerosumfree.

Example 1. The following dioids are to be compared in the text:

1. The Boolean lattice $\mathbb{B} \equiv 2 \equiv\langle\{0,1\}, \vee, \wedge, 0,1\rangle$

2. The fuzzy algebra $\rrbracket_{\max , \min } \equiv\langle[0,1], \max , \min , 0,1\rangle[18]$

3. The tropical semiring $\mathbb{N}_{\min ,+} \equiv\langle\mathbb{N} \cup\{0, \infty\}$, min, $+, \infty, 0\rangle$

4. The max-plus algebra $\mathbb{R}_{\max ,+} \equiv\langle\mathbb{R} \cup\{-\infty\}$, max, $+,-\infty, 0\rangle$ [19]

5. The optimization algebra, $\mathbb{R}_{\min ,+} \equiv\langle\mathbb{R} \cup\{\infty\}, \min ,+, \infty, 0\rangle[19]$

6. The max-times semiring, $\mathbb{R}_{\max , x} \equiv\left\langle\mathbb{R}_{\mathbb{0}}^{+} \cup\{\infty\}\right.$, $\left.\max , \times, 0,1\right\rangle[14]$

7. The fuzzy max-times algebra, $\square_{\max , x} \equiv\langle[0,1], \max , \times, 0,1\rangle[14]$

Their relationships can be gleaned from Fig. 1.

A big class of dioids is that of (additively) idempotent semirings. An idempotent semiring $\mathcal{D}$ is a semiring whose additive structure $\langle D, \oplus, \epsilon\rangle$ is an idempotent semigroup, that is, $\forall a \in D, a \oplus a=a$. Idempotent semirings are all canonically-ordered and, if commutative, they are already $\vee$-semilattices (read sup-or join-semilattice), whose operation is compatible with the canonical order $a \oplus b=a \vee b$ and selects the lowest upper bound, supremum or join [14, Chap. 1, Theorems $1 \& 2$ ]. The simple semirings, or commutative inclines, a useful generalization of the fuzzy algebras, are idempotent semirings whose unit is also the maximum in the order $e=\top[6, \mathrm{p}$. 4]. Note that an idempotent semiring is selective [14] or extremal [6] if the argument where the addition is attained can be selected. (Commutative) selective semirings are all totally-ordered [14, Chap. 1, 3.4.7], [6, p. 228], like $\mathbb{B}, \mathbb{R}_{\max ,+}$ or $\mathbb{1}_{\max , x}$.

In this paper we are going to delve into the idiosyncratic character of some idempotent semirings stemming from their multiplicative structure. A commutative semiring is one whose multiplicative structure is commutative, a multiplicatively-idempotent semiring one whose multiplicative structure is a (commutative) idempotent monoid, 


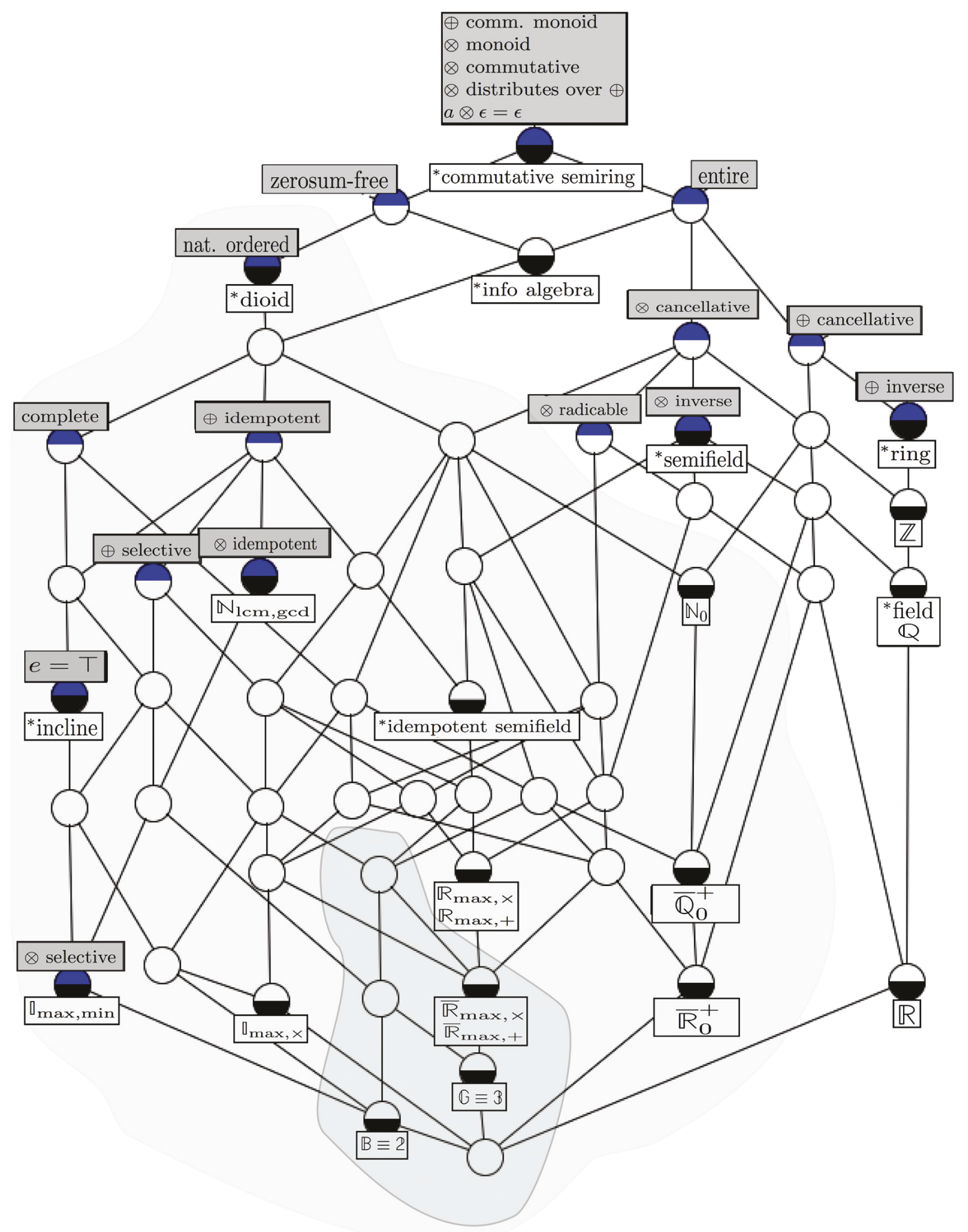

Fig. 1. Concept lattice of a choice of abstract (leading asterisk, white label) and concrete (white label) commutative semirings and their properties (grey label) mentioned in the text. Each node is a concept of Abstract Algebra: its properties are obtained from the grey labels in nodes upwards, and its structures from the white labels in nodes downwards. The picture is related to the chosen sets of properties and algebras and does not fully reflect the structure of the class of semirings. We have chose to highlight dioids and, within them, complete idempotent semifields. 
and a division semiring is one whose multiplicative structure $\left\langle K \backslash\left\{\epsilon, \otimes, e,{ }^{-1}\right\rangle\right.$ is a group, that is, there is an operation, $.^{-1}: S \backslash\{\epsilon\} \rightarrow S \backslash\{\epsilon\}$ such that $\forall a \in S, a \otimes a^{-1}=a^{-1} \otimes a=e$. A radicable [14] or algebraically complete [6] semiring is one in which equation $a^{b}=c$ can be solved for $a$. A semifield is a commutative division semiring [6], so an idempotent semifield is an idempotent semiring $\mathcal{K}$ whose multiplicative structure is a commutative group. For semifields we have $(a \otimes b)^{-1}=a^{-1} \otimes b^{-1}$. Both $\mathbb{R}_{\max ,+}$ and $\mathbb{R}_{\min ,+}$, our main interest in this paper, are idempotent semifields with the same multiplicative inverse $\cdot^{-1}:=-$.

A nonzero element $a$ of a semiring $\mathcal{S}$ is a left zero divisor iff there exists a nonzero element $b \in \mathcal{S}$ such that $b \otimes a=\epsilon$. Right zero divisors are defined similarly. It is a zero divisor iff it is either a left or a right zero divisor. A semiring with no zero divisors is zero-divisor free or entire. Entire zerosum-free semirings are also called information algebras and will prove important in our description. Semifields are all entire, whence idempotent semifields are all information algebras.

All of the above examples are idempotent semirings, but $\mathbb{B}, \mathbb{R}_{\max ,+}$ and $\mathbb{R}_{\min ,+}$ (both with inverse $\cdot^{-1}:=-\cdot$ ) and $\mathbb{R}_{\max , \times}$ (with the usual multiplicative inverse) are also idempotent semifields. Notice that in a dioid with a multiplicative group structure the bottom cannot have an inverse since $a \otimes \epsilon=\epsilon \neq e$, for $a \in S$. Therefore, they are all incomplete, lacking a top element, except for $\mathbb{B} \equiv 2$.

Recall that the canonical order turns idempotent semirings into sup-semilattices $\langle D, \vee\rangle$ with the supremum defined as $a \vee b=a \oplus b$. A $\wedge$-semilattice (read inf-or meet-semilattice) is likewise defined to select the greatest lower bound, or infimum in the order. A lattice is an ordered set $\mathcal{L}=\langle L, \leq\rangle$ which is at the same time an algebra $\mathcal{L}=\langle L, \vee, \wedge\rangle$ where $\langle L, \vee\rangle$ is a $\vee$-semilattice and $\langle L, \wedge\rangle$ a $\wedge$-semilattice, and the absorption laws hold: $a \vee(a \wedge b)=a$ and $a \wedge(a \vee b)=a$. In general, a semiring is not a lattice. But in an idempotent semifield the formula for the infimum of two elements was already given by Dedekind [14]: the meet law is $a \wedge b=a \otimes(a \oplus b)^{-1} \otimes b$. Thus, idempotent semifields are lattices in their canonical order $\langle K, \vee, \wedge\rangle$, with bottom element $\perp=\epsilon$.

Henceforth $\mathcal{S}$ will denote a generic semiring, $\mathcal{D}$ a dioid, and $\mathcal{K}$ an idempotent semifield. To emphasize when a semiring is complete we will use an overline, as in $\overline{\mathcal{K}}$. For further information about semirings consult $[6,14]$.

\subsection{Complete idempotent semifields and semimodules}

A complete semiring $\mathcal{S}$ [17] is a semiring where for every (possibly infinite) family of elements $\left\{a_{i}\right\}_{i \in I} \subseteq S$ we can define an element $\sum_{i \in I} a_{i} \in S$ such that

1. if $I=\varnothing$, then $\sum_{i \in I} a_{i}=\epsilon$,

2. if $I=\{1 \ldots n\}$, then $\sum_{i \in I} a_{i}=a_{1} \oplus \cdots \oplus a_{n}$,

3. if $b \in S$, then $b \otimes\left(\sum_{i \in I} a_{i}\right)=\sum_{i \in I} b \otimes a_{i}$ and $\left(\sum_{i \in I} a_{i}\right) \otimes b=\sum_{i \in I} a_{i} \otimes b$, and

4. if $\left\{I_{j}\right\}_{j \in J}$ is a partition of $I$, then $\sum_{i \in I} a_{i}=\sum_{j \in J}\left(\sum_{i \in I_{j}} a_{i}\right)$.

If $I$ is countable in the definitions above, then $\mathcal{S}$ is countably complete and already zerosumfree [6, Prop. 22.28].

The existence of the following elements is crucial for our purposes after Theorem 2.4: In a semiring $\mathcal{S}$ for each $a \in S$, define

$$
a^{* k}=e \oplus a \oplus a^{2} \oplus \ldots \oplus a^{k}=\sum_{i=0}^{k} a^{i} \quad a^{+k}=a \oplus a^{2} \oplus \ldots \oplus a^{k}=\sum_{i=1}^{k} a^{i}
$$

In complete semirings, the Kleene star is the sum $a^{*}=\sum_{k \in \mathbb{N}_{0}} a^{k}$. Likewise the Kleene plus is the sum $a^{+}=\sum_{k \in \mathbb{N}} a^{k}$, when it exists. Since $a^{* k}=e \oplus a^{+k}$ and $a^{+k}=a \otimes a^{*(k-1)}=a^{*(k-1)} \otimes a$ the existence of one of these elements entails the existence of the other:

$$
a^{*}=e \oplus a^{+} \quad a^{+}=a \otimes a^{*}=a^{*} \otimes a .
$$

To investigate completion issues, call an element in a semiring $a \in S$ infinite iff $a \oplus b=a$ for all $b \in S$, and strongly infinite if also $a \otimes b=a=b \otimes a[6]$. If $\overline{\mathcal{S}}$ is a complete semiring, then it has a (necessarily unique) infinite element [6, Prop. 22.27].

Complete semirings with strongly infinite elements can be created on demand: 
Example 2 (Completion with strongly infinite element). (See [6, p. 250, Chap. 22] and [20, p. 16].) If $\mathcal{S}$ is an entire zerosumfree semiring, it can be endowed with a unique strongly infinite element: for $\infty \notin S$ consider $\bar{S}=S \cup\{\infty\}$ and extend $\mathcal{S}=\langle S, \oplus, \otimes, \epsilon, e\rangle$ to $\overline{\mathcal{S}}=\langle S \cup\{\infty\}, \oplus, \otimes, \epsilon, e\rangle$ by $b \oplus \infty=\infty \oplus b=\infty \oplus \infty=\infty$ for all $b \in S$, $b \otimes \infty=\infty \otimes b=\infty \otimes \infty=\infty$ for all $b \in S /\{\epsilon\}$ and $\epsilon \otimes \infty=\infty \otimes \epsilon=\epsilon$. Clearly $\infty$ is the unique infinite element and it is strongly infinite by definition.

We have:

Proposition 2.1. (See [6, 22.32].) If $\mathcal{S}$ is a (commutative) entire zerosumfree semiring, its strong infinite completion $\overline{\mathcal{S}}$ is a (commutative) complete entire zerosumfree semiring.

Recall that on any semiring $\mathcal{S}$, left and right multiplications can be defined: $\mathrm{L}_{a}: S \rightarrow S, b \mapsto \mathrm{L}_{a}(b)=a b$, and $\mathrm{R}_{a}: S \rightarrow S, b \mapsto \mathrm{R}_{a}(b)=b a$. A dioid $\mathcal{D}$ is complete, if it is complete as a semiring, further complete as a naturally ordered set $\langle D, \preccurlyeq\rangle$ (see Section 2.1) and left $\left(\mathrm{L}_{a}\right)$ and right $\left(\mathrm{R}_{a}\right)$ multiplications are lower semicontinuous, that is, join-preserving. In such cases, the top of the dioid is the unique infinite element $\top=\sum_{a \in D} a$, whence $\top=\top \oplus a$ for all $a \in D$, but $\top \otimes \perp=\perp$. Note that in complete dioids an adequate notion of topology can be defined, the sup-topology, where infinite summation can be defined in terms of suprema, $\sum_{i \in I} a_{i}=\bigvee_{i \in I} a_{i}$.

An element $a \in D$ of a dioid is $k$-stable iff for $k \geq 0, a^{*(k+1)}=a^{* k}[14$, p. 97]. The following results are crucial later on:

\section{Proposition 2.2.}

1. If $\mathcal{D}$ is a dioid then

(a) if a is $k$-stable, then $a^{*}=a^{* k}$,

(b) $a \preccurlyeq a^{+k} \preccurlyeq a^{* k}$,

(c) for $a \preccurlyeq b$ we have $a^{+k} \preccurlyeq b^{+k}$ and $a^{* k} \preccurlyeq b^{* k}$,

(d) $\perp=\perp^{+} \prec \perp^{*}=e$.

2. Furthermore, if $\overline{\mathcal{D}}$ is a complete dioid, then

(a) $a \preccurlyeq a^{+} \preccurlyeq a^{*}$,

(b) for $a \preccurlyeq b$ we have $a^{+} \preccurlyeq b^{+}$and $a^{*} \preccurlyeq b^{*}$,

(c) for $e \preccurlyeq b$ we have $b^{+}=b^{*}$,

(d) $e^{+}=e^{*}$ and $\mathrm{T}=\mathrm{T}^{+}=\mathrm{T}^{*}$.

3. Furthermore, if $\overline{\mathcal{D}}$ is a complete multiplicatively-cancellative dioid then for $b \in D$ such that $e \prec b \prec \top$ we have $b^{*}=\top$.

4. Furthermore, if $\overline{\mathcal{D}}$ is a complete idempotent dioid then

(a) $\left(a^{*}\right)^{2}=a^{*},\left(a^{*}\right)^{*}=a^{*}, a^{* k}=(e \oplus a)^{k}$ and even $a^{*}=(e \oplus a)^{+}$,

(b) for $a \in D$ such that $\perp \preccurlyeq a \prec e$ we have $a=a^{+} \prec a^{*}=e$ and $e=e^{+}=e^{*}$.

5. Furthermore, if $\overline{\mathcal{D}}$ is a complete multiplicatively-cancellative idempotent dioid then if $a, b \in D$ exist such that $\perp \prec a \prec e \prec b \prec T$ we have

$$
\perp=\perp^{+} \prec a=a^{+} \prec \perp^{*}=a^{*}=e=e^{+}=e^{*} \prec b^{+}=b^{*}=\top=\top^{+}=\top^{*}
$$

Proof. If $a$ is $k$-stable, by induction we may prove $a^{*(k+r)}=a^{* k} \forall r \geq 0$, whence $1 \mathrm{a}$. From $a^{* k}=a^{+k} \oplus e$, claim $1 \mathrm{~b}$ follows. For $a \preccurlyeq b$ by the compatibility of the product it is not difficult to obtain $a^{k} \preccurlyeq b^{k}$. By the compatibility of the sum of all power inequalities claim 1c follows. Finally, $\perp^{*}=\perp \otimes \perp^{*} \oplus e=e$, whence $\perp^{+}=\perp^{*} \otimes \perp=\perp$.

Since in complete dioids Kleene's stars exist, claim 2a follows from (2). With countable summation in claim 1c we obtain $2 \mathrm{~b}$. If $e \preccurlyeq b$, by the compatibility of multiplication $b^{*} \preccurlyeq b \otimes b^{*}=b^{+}$whence 2 c. Claim $2 \mathrm{~d}$ follows with $\top^{+}=\top \oplus \sum_{i \geq 2} \top^{k}=\top$.

For claim 3, if $e \prec b \prec \top$ then by claims $2 \mathrm{~b}$ and $2 \mathrm{c}, b^{+}=b^{*} \preccurlyeq \top$. On multiplicatively-cancellative partially-ordered semirings the following strong law of compatibility holds [20, Chap. III, Lemma 2.4]: if $a<b$ then for all $c \in D, a \otimes c<b \otimes c$ and $c \otimes a<c \otimes b$. A fortiori, the law holds on multiplicatively-cancellative dioids, so for multiplicatively-cancellable $b^{*} \prec \top$ from $e \prec b$ we get $b^{*} \prec b^{*} \otimes b=b^{+}$, a contradiction, so $b^{*}=\top$. 
Claim $4 \mathrm{a}$ is well-known [21]. For claim $4 \mathrm{~b}$, since in an (additively-)idempotent dioid we have $a \oplus b=b \Leftrightarrow a \preccurlyeq b$, so from $a \preccurlyeq e$ we have $a^{k} \preccurlyeq a^{k-1}$ whence $a^{* k}=a^{k} \oplus \ldots \oplus a \oplus e=e$ and $a^{+k}=a^{k} \oplus \ldots \oplus a=a$. Since the semirings are complete, the countably infinite summations exist. Claim 5 is just a corollary of the rest of claims.

All the above can be seen instantiated in:

\section{Example 3.}

1. In $\mathbb{B}$ we have $0^{*}=1^{*}=1$, hence 1 is (vacuously) strongly infinite.

2. In the schedule algebra $\mathbb{R}_{\max ,+}$ we have $a^{k}:=k \cdot a$ so $e \oplus a^{k}:=\max (0, k \cdot a)$. That is, for $a \leq 0, e \oplus a^{k}=e$ and $a^{*}:=0$. On the other hand, for $b>0, e \oplus b^{k}=b^{k}$ and such elements do not have a star, hence $\mathbb{R}_{\max ,+}$ is incomplete. When we complete $\overline{\mathbb{R}}_{\max ,+}$ with $\top:=\infty$, for $b>e$ we have $b^{*}=b^{+}=\top$.

In general $\mathrm{T}$ is not strongly infinite, but for the construction in Example 2 we have:

Proposition 2.3. (See [6, Prop. 22.14].) If $\mathcal{D}$ is a totally-ordered, entire positive semiring then it can be (countably) completed to $\overline{\mathcal{D}}$ with $T=\sum D$ a strongly infinite element.

Proof. Use $\top=\infty \notin D$ in the completion, with the natural order in $\mathcal{D}$ extended by $a \prec \top$ for all $a \in D$, and let $\sum_{i \in I} a_{i}=\sup \left\{\sum a_{i} \mid J \subseteq I, J\right.$ finite $\}$, whence $T=\sum D$.

Note that even already complete semirings can be extended in this way. For instance, $\mathbb{B}$ can be extended to $B$ below. There are plenty of completable totally-ordered semirings, like entire selective semirings [6, Chap. 20]. In the following, by completed semirings we mean those in the completion of Example 2, and by completed (entire, totally-ordered) dioids we mean those of Proposition 2.3.

A fortiori, selective semifields can all be completed, as, for instance, the (initially incomplete) maxplus and minplus semifields in Example 3 [22-25]:

1. the completed Minplus semifield, $\overline{\mathbb{R}}_{\min ,+}=\langle\mathbb{R} \cup\{-\infty, \infty\}$, min, $\dot{+}, \infty, 0,-\infty\rangle$,

2. the completed Maxplus semifield, $\overline{\mathbb{R}}_{\max ,+}=\langle\mathbb{R} \cup\{-\infty, \infty\}, \max ,+,-\infty, 0, \infty\rangle$.

These two completions are actually inverses $\overline{\mathbb{R}}_{\min ,+}=\overline{\mathbb{R}}_{\max ,+}^{-1}$ and order-dual [22]. Indeed they are better jointly called the max-min-plus semiring $\overline{\mathbb{R}}_{\max ,+}^{\min , \dot{+}}$. We have $-\infty+\infty=-\infty$ and $-\infty \dot{+} \infty=\infty$, which solves several issues in dealing with the separately completed dioids. ${ }^{1}$ This was first recorded as a blog, a bounded, lattice-ordered group [27, §4.1], although the name did not catch, and would be called a bounded $\ell$-group nowadays. The lattice $\mathbb{B}$ can be embedded in any bounded $\ell$-group, by restricting the carrier set to $\{\perp, \top\}$. The boolean operations would then be implemented as $\oplus$ and $\otimes$ restricted to such set. A richer structure is the 3 -element bounded $\ell$-group $B=\langle\{\perp, e, \top\}, \oplus, \dot{\oplus}, \otimes, \dot{\otimes}, \perp, \dot{e}, \top\rangle$. Such structure is therefore isomorphically embedded into any completed, naturally-ordered semifield by the restriction of its operations to the carrier set $\{\perp, e, \top\}$. This is the only bounded $\ell$-group having a finite number of elements [27, Propos. 4.6-4.9], and will prove crucial for the representation of certain lattices related to eigenspaces of completed idempotent semifields.

In this context, a semimodule over a semiring, is the analogue of a module over a ring $[6,17,28]$ : a right $\mathcal{S}$-semimodule is an additive commutative monoid $\mathcal{X}=\langle X, \oplus, \epsilon \mathcal{X}\rangle$ endowed with a right action $(x, \lambda) \mapsto x \odot \lambda$ such that $\forall \lambda, \mu \in S, x, x^{\prime} \in X$. Following the convention of dropping the symbols for the scalar action and semiring multiplication we have:

\footnotetext{
1 In this paper we propound the use of dotted $\otimes$ and $\oplus$ signs for the operations in either semiring: they are more coherent with Moreau's notation [22], which has the precedence, and is reminiscent of [26] for max-times. Besides, the notation using an apostrophe for the min-related operations in [27]—clearly downplaying the min-plus semiring — seems to have been prompted by obsolete typesetting technology.
} 


$$
\begin{aligned}
& x(\lambda \mu)=(x \lambda) \mu \quad x \epsilon=\epsilon \mathcal{X} \\
& \left(x \oplus x^{\prime}\right) \lambda=x \lambda \oplus x^{\prime} \lambda \quad x e=x
\end{aligned}
$$

The definition of a left $\mathcal{S}$-semimodule $\mathcal{Y}$ follows the same pattern with the help of a left action, $(x, \lambda) \mapsto \lambda \odot x$ and similar axioms. An $(\mathcal{R}, \mathcal{S})$-semimodule is a set $M$ endowed with left $\mathcal{R}$-semimodule and a right $\mathcal{S}$-semimodule structures, and an $(\mathcal{R}, \mathcal{S})$-bisemimodule an $(\mathcal{R}, \mathcal{S})$-semimodule such that the left and right actions commute. Column spaces $\mathcal{S}^{n \times 1}$ are $\left(\mathcal{S}^{n \times n}, \mathcal{S}\right)$-bisemimodules, and row spaces $\left(\mathcal{S}, \mathcal{S}^{n \times n}\right)$-bisemimodules.

In a semimodule $\mathcal{X}$ over a semifield $\mathcal{K}$ one can define an element-wise inversion operation $\cdot^{-1}: X \rightarrow X$, $x \mapsto x^{-1}$ such that $\left(x^{-1}\right)_{i}=x_{i}^{-1}$. If the semifield is also a dioid, then the "inverse" semimodule is the order dual $\mathcal{X}^{-1} \cong\left\langle X, \leq^{\mathrm{d}}\right\rangle$.

Example 4. Semimodules over $\overline{\mathbb{R}}_{\max ,+}$ have inverses over $\overline{\mathbb{R}}_{\min ,+}$ and vice versa. In particular $\left(\overline{\mathbb{R}}_{\max ,+}\right)^{-1}=\overline{\mathbb{R}}_{\min ,+}$, and dually.

A complete semimodule [6] is also a complete lattice, with join and meet operations fulfilling $v_{1} \leq v_{2} \Longleftrightarrow v_{1} \vee$ $v_{2}=v_{2} \Longleftrightarrow v_{1} \wedge v_{2}=v_{2}$. In the case of semimodules over complete dioids with a multiplicative group structure one has $v_{1} \wedge v_{2}=\left(v_{1}^{-1} \vee v_{2}^{-1}\right)^{-1}$ à la Boole. For $\overline{\mathbb{R}}_{\max ,+}$, it is $v_{1} \wedge v_{2}=v_{1} \dot{\oplus} v_{2}=\left(v_{1}^{-1} \oplus v_{1}^{-1}\right)^{-1}=\min \left(v_{1}, v_{2}\right)$.

For $n, p \in \mathbb{N}$, the semimodule of finite matrices $\mathcal{M}_{n \times p}(\mathcal{S})=\left\langle S^{n \times p}, \oplus, \mathcal{E}\right\rangle$ is an $\left(\mathcal{M}_{n}(\mathcal{S}), \mathcal{M}_{p}(\mathcal{S})\right.$ )-bisemimodule, with matrix multiplication-like left and right actions and entry-wise addition. Special cases of it are the bisemimodules of column vectors $\mathcal{M}_{p \times 1}(\mathcal{S})$ and row vectors $\mathcal{M}_{1 \times n}(\mathcal{S})$. In the following we systematically equate left (resp. right) $\mathcal{S}$-semimodules and row (resp. column) semimodules over $\mathcal{S}$.

\subsection{The spectral problem in semirings}

Given a square matrix $A \in S^{n \times n}$ the right (left) eigenproblem is the task of finding the right eigenvectors $v \in S^{n \times 1}$ and right eigenvalues $\rho \in S$ (respectively left eigenvectors $u \in S^{1 \times n}$ and left eigenvalues $\lambda \in S$ ) satisfying:

$$
u \otimes A=\lambda \otimes u \quad A \otimes v=v \otimes \rho
$$

The left and right eigenspaces $-\mathcal{U}_{\lambda}(A)$ and $\mathcal{V}_{\rho}(A)$ - and spectra- $\Lambda(A)$ and $\mathrm{P}(A)$ - are the sets of solutions:

$$
\begin{aligned}
& \mathcal{U}_{\lambda}(A)=\left\{u \in S^{1 \times n} \mid u \otimes A=\lambda \otimes u\right\} \quad \mathcal{V}_{\rho}(A)=\left\{v \in S^{n \times 1} \mid A \otimes v=v \otimes \rho\right\} \\
& \Lambda(A)=\left\{\lambda \in S \mid \mathcal{U}_{\lambda}(A) \neq\left\{\epsilon^{n}\right\}\right\} \quad \mathrm{P}(A)=\left\{\rho \in S \mid \mathcal{V}_{\rho}(A) \neq\left\{\epsilon^{n}\right\}\right\}
\end{aligned}
$$

Since $\Lambda(A)=\mathrm{P}\left(A^{\mathrm{T}}\right)$ and $\mathcal{U}_{\lambda}(A)=\mathcal{V}_{\lambda}\left(A^{\mathrm{T}}\right)$, from now on we will omit references to left eigenvalues, eigenvectors and spectra, unless we want to emphasize differences.

Regarding the structure of right eigenspaces, it is well-known that they are right subsemimodules of $\mathcal{S}^{n \times 1}[6$, p. 219], [29, \$4.1.1]. When an eigenspace can be finitely generated, it will be convenient to define it as the span of some column eigenvectors gathered in a matrix $V \in \mathcal{S}^{n \times m}$ as $\langle V\rangle_{\mathcal{S}}=\left\{V \otimes z \mid z \in \mathcal{S}^{m \times 1}\right\}$.

Notice that the spectral theories of rings and zerosumfree semirings are deeply different even at first glance: the well-known techniques for $\mathbb{R}_{+, x}$ or $\mathbb{C}_{+, x}$ are totally different to the less-known techniques like the Perron-Frobenius theory for $\mathbb{R}_{+, x}^{+}=\left\langle\mathbb{R}_{0}^{+},+, \times, 0,1\right\rangle$-widely used in search engine technology.

With so little structure it might seem hard to solve (3). Readily available techniques are combinatorial in nature and a very generic solution exists based on the following recurring concept. For a matrix over a semiring $A \in S^{n \times n}$ consider the sum $A^{* k}=I \oplus A \oplus A^{2} \oplus \cdots \oplus A^{k}$, where addition and multiplication over matrices are intuitively obtained from those of the underlying semiring, $A^{k}$ represents a product of $k$ factors and $I$ is the neutral element for matrix multiplication (cf. Section 2.1). The Kleene star of $A$ is $A^{*}=\sum_{k \in \mathbb{N}_{0}} A^{k}$ and the Kleene plus of $A$ is $A^{+}=A \otimes A^{*}$. Most of the results on eigenvalues and eigenvectors in this paper stem from the following fact:

Proposition 2.4. (See Gondran and Minoux, Theorem 1 [13,14].) Let $A \in \mathcal{S}^{n \times n}$. If $A^{*}$ exists, the following two conditions are equivalent:

1. $A_{. i}^{+} \otimes \mu=A_{. i}^{*} \otimes \mu$ for some $i \in\{1 \ldots n\}$, and $\mu \in S$.

2. $A_{. i}^{+} \otimes \mu\left(\right.$ and $\left.A_{. i}^{*} \otimes \mu\right)$ is an eigenvector of $A$ for $e, A_{. i}^{+} \otimes \mu \in \mathcal{V}_{e}(A)$. 
Proof. $(1 \Rightarrow 2)$ If $A_{\cdot i}^{*} \otimes \mu=A_{\cdot i}^{+} \otimes \mu$, since $A \otimes A^{*}=A^{+}$, then we have $A \otimes A_{. i}^{*} \otimes \mu=A_{. i}^{*} \otimes \mu$ which proves that $A_{. i}^{*} \otimes \mu \in \mathcal{V}_{e}(A)$ (hence $\left.A_{\cdot i}^{+} \otimes \mu\right) .(2 \Rightarrow 1)$ Assume $A_{\cdot i}^{*} \otimes \mu \in \mathcal{V}_{e}(A)$. Then $A \otimes A_{\cdot i}^{*} \otimes \mu=A_{. i}^{*} \otimes \mu$. On the other hand, since $A \otimes A^{*}=A^{+}$, we have $A \otimes A_{\cdot i}^{*} \otimes \mu=A_{\cdot i}^{+} \otimes \mu$ hence, $A_{\cdot i}^{*} \otimes \mu=A_{\cdot i}^{+} \otimes \mu$.

Depending on the properties of the semiring, transitive closures may be easy to calculate [30] or non-existent. As transitive closures always exist in complete dioids, this will be our natural upper bound in the lattice of semirings (see Fig. 1).

However, although a number of spectral results exist for information algebras—entire zerosumfree semirings-and dioids, we prefer to adopt idempotent semifields as the basic level in the lattice of structures in Fig. 1. Unfortunately, this means most of our results will not be available in inclines or non-idempotent semifields, e.g. $\mathbb{R}_{0}^{+}[14]$. So that no connection is lost to these other methods, when results are general enough we will state them in the highest possible level in the lattice of semirings. For instance, a right semimodule $\mathcal{X}$ over an idempotent semiring $\mathcal{D}$ inherits the idempotent law: $\forall x \in X, x \oplus x=x$, which induces a natural order on the semimodule

$$
\forall x, x^{\prime} \in X, \quad x \preccurlyeq x^{\prime} \Longleftrightarrow x \oplus x^{\prime}=x^{\prime}
$$

whereby it is already a $\vee$-semilattice with $x \vee x^{\prime}=x \oplus x^{\prime}$ and $\epsilon \mathcal{X}$ its minimum, whence:

Corollary 2.5. Let $A \in \mathcal{D}^{n \times n}$ be a matrix with entries in a commutative idempotent semiring $\mathcal{D}$. For all eigenvalues $\rho \in \mathrm{P}(A), \mathcal{V}_{\rho}(A)$ is a $\vee$-semilattice with bottom $\perp^{n}$.

\subsection{Square matrices over semirings and their cryptomorphisms}

All of the problems in the previous section can be solved by considering different interpretations for matrices over a semiring: namely, merely as arrangement of numbers, as linear forms, as relations or as directed graphs.

If $\mathcal{S}$ is a semiring, $\mathcal{M}_{n}(\mathcal{S})=\left\langle S^{n \times n}, \oplus, \otimes, \mathcal{E}, I\right\rangle$ is the semiring of (square) matrices over $\mathcal{S}$ with $S^{n \times n}$ denoting the set of square matrices of order $n$, matrix operations $(A \oplus B)_{i j}=A_{i j} \oplus B_{i j}, 0 \leq i, j \leq n$ and $(A \otimes B)_{i j}=$ $\sum_{k=1}^{n} A_{i k} \otimes B_{k j}, 0 \leq i, j \leq n$, null matrix $\mathcal{E}, \mathcal{E}_{i j}=\epsilon, 0 \leq i, j \leq n$ and unit matrix $I, I_{i i}=e, 0 \leq i \leq n, I_{i j}=\epsilon$, $0 \leq i, j \leq n, i \neq j$. Such semirings are not commutative in general even if $\mathcal{S}$ is, except for $\mathcal{M}_{1}(\mathcal{S})=\mathcal{S}$. They are idempotent and complete if $\mathcal{S}$ is.

If $\overline{\mathcal{K}}$ is a completed semifield, then matrix multiplications for conformant $A, B$ are:

$$
(A \otimes B)_{i j}=\sum_{k=1}^{n} A_{i k} \otimes B_{k j} \quad(A \dot{\otimes} B)_{i j}=\sum_{k=1}^{n} A_{i k} \dot{\otimes} B_{k j}
$$

\subsubsection{Matrices as number arrays}

From its definition, $A \in \mathcal{M}_{n}(\mathcal{S})$ carries implicitly a set of indices $\overline{\mathbf{n}}=\{1, \ldots, n\} .{ }^{2}$ Given subsets of indices $\alpha, \beta \subseteq \overline{\mathbf{n}}$ we denote by $A_{\alpha \beta}=A(\alpha, \beta)$ the submatrix of $A$ selected by the indices in classes $\alpha, \beta$. It is convenient to denote by $A_{\cdot j}=A(\overline{\mathbf{n}},\{j\})$ (resp. $\left.A_{j}=A(\{j\}, \overline{\mathbf{n}})\right)$ with $j \in \overline{\mathbf{n}}$ the $j$-th column (resp. row) of a matrix.

Given a linear ordering of the indices $\sigma$, its permutation matrix is $P(\sigma)=I(\overline{\mathbf{n}}, \sigma)$. The permutation of the columns of $A$ as in $\sigma$ is denoted by $A \otimes P(\sigma)=A(\overline{\mathbf{n}}, \sigma)$, and the permutation of its rows is $P^{\mathrm{T}}(\sigma) \otimes A=A(\sigma, \overline{\mathbf{n}})$. For $A, B \in \mathcal{M}_{n}(\mathcal{S})$ we say that $B$ is permutationally equivalent to $A, A \cong B$, if there exists a permutation (matrix) $P(\sigma)$ such that $B=P^{\mathrm{T}}(\sigma) \otimes A \otimes P(\sigma)=A(\sigma, \sigma)$. The eigenspaces of permutationally equivalent matrices can be related:

Lemma 2.6. (See $\left[29\right.$, Prop. 4.1.3].) Let $A, B \in \mathcal{M}_{n}(\mathcal{S})$ and $B=P^{\mathrm{T}} \otimes A \otimes P$, where $P$ is a permutation matrix. Then there is a bijection between $\mathcal{V}(A)$ and $\mathcal{V}(B)$ described by $\mathcal{V}(B)=\left\{P^{\mathrm{T}} \otimes v \mid v \in \mathcal{V}(A)\right\}$, and likewise for left spectra, mutatis mutandis.

Call a matrix reducible if $n \geq 2$ and for some integer $r$ with $1 \leq r \leq n-1$, there exists an $r \times(n-r)$ zero submatrix that does not meet the main diagonal of $A$-equivalently iff it is permutationally equivalent to a blocked form as in (6) - and irreducible otherwise.

\footnotetext{
2 The notation $\overline{\mathbf{n}}$ is chosen to resemble that of antichains.
} 


$$
P^{\mathrm{T}} \otimes A \otimes P=\left[\begin{array}{cc}
A_{\alpha \alpha} & A_{\alpha \beta} \\
\cdot & A_{\beta \beta}
\end{array}\right]
$$

Proposition 2.7. Let $A \in \mathcal{M}_{n}(\mathcal{S})$ be a matrix over a semiring. The following are equivalent:

1. A is irreducible.

2. For each pair $\{i, j\} \subseteq \overline{\mathbf{n}}$, there are paths from $i$ to $j$ and from $j$ to $i$.

3. If further $\mathcal{S}$ is zerosumfree, for each pair $\{i, j\} \subseteq \overline{\mathbf{n}}, A_{i j}^{+} \neq \epsilon$ and $A_{j i}^{+} \neq \epsilon$.

Corollary 2.8. If $A \in \mathcal{M}_{n}(\mathcal{S})$ is an irreducible matrix over a semiring, then none of its rows or columns is null.

Properties maintained modulo permutation equivalence are weak combinatorial invariants of a matrix:

Proposition 2.9. (See [31, \$27.1] [29, Prop. 4.1.3].) Weak combinatorial invariants of a matrix are:

1. The multiset of elements and the number of zeros in its diagonal.

2. The composition of its spectra.

3. Whether it is reducible or irreducible.

4. The cycles and cycle weights of its induced network (see Section 2.4.3).

\subsubsection{Matrices as relations}

Let $A \in \mathcal{M}_{n}(\mathcal{S})$ be a semiring-valued matrix:

1. the transpose of $A$ is the matrix $\left(A^{\mathrm{T}}\right)_{i j}=A_{j i}$. Transposition is an involution,

2. the reflexive closure of $A$ is $r(A)=A \oplus I_{n}$,

3. the symmetric closure of $A$ is $s(A)=A \oplus A^{\mathrm{T}}$,

4. the transitive closure [14]—also Kleene plus [21] or metric matrix [27]—of $A$ is the matrix $A^{+}=\sum_{k \in \mathbb{N}} A^{i}$ when such sum exists,

5. the transitive-reflexive closure-also Kleene-star or quasi-inverse-of $A$ is the matrix $A^{*}=\sum_{k \in \mathbb{N}_{0}} A^{i}$ when the sum exists.

Note that transitive(-reflexive) closures differ, at most, in their diagonals $A_{i i}^{*}=e \oplus A_{i i}^{+}, i \in \overline{\mathbf{n}}$. We will rely in Proposition 2.2 , specially claim 4 a, to calculate them efficiently.

\subsubsection{Matrices as networks with weights in a semiring}

A digraph (or directed graph), ${ }^{3}$ is a pair $G=(V, E)$, with $V$ a set of vertices and $E \subseteq V \times V$ a set of arcs (directed edges), ordered pairs of vertices, such that for every $i, j \in V$ there is at most one arc $(i, j) \in E$. Let a path in $G$ be a sequence of $\operatorname{arcs} w=\left(i_{0}, i_{1}\right),\left(i_{1}, i_{2}\right),\left(i_{2}, i_{3}\right), \ldots,\left(i_{k-1}, i_{k}\right)$, pairwise sharing a vertex; a cycle a path with $i_{o}=i_{k}$; and a loop a cycle composed of the single arc $(i, i)$. Then let an elementary path be a path none of whose vertices is repeated, except possibly for $i_{o}=i_{k}$, and likewise for elementary cycles.

For every pair of vertices $i, j \in V$ and $k \in \mathbb{N}$, let $\Pi_{G}^{k}(i, j)$ be the set of paths of length $k$ from $i$ to $j ; \Pi_{G}^{+(k)}(i, j)=$ $\bigcup_{l=1}^{k} \Pi_{G}^{l}(i, j)$ be the set of paths of length up to $k$, and $\Pi_{G}^{+}(i, j)=\bigcup_{k \in \mathbb{N}} \Pi_{G}^{k}(i, j)$ be the (possibly empty) set of paths of any length from $i$ to $j$. Similarly, let $C_{G}^{k}(i)$ be the set of cycles of length $k$ through vertex $i$ and $C_{G}^{+}(i)=\bigcup_{k \in \mathbb{N}} C_{G}^{k}(i)$ be the set of cycles of all possible lengths through vertex $i$. The set of elementary paths from $i$ to $j$ and cycles through $i$ are $\Pi_{G}^{e}(i, j)$ and $C_{G}^{e}(i)$, respectively.

If $\mathcal{S}$ is a semiring, an $\mathcal{S}$-network or $\mathcal{S}$-weighted digraph $N_{\mathcal{S}}=(V, E, w)$ is an underlying digraph $G_{N}=(V, E)$ together with an $S$-valued weight (or cost) function $w: V \times V \rightarrow S \backslash \epsilon$ on the set of arcs. If $\mathcal{S} \equiv \mathbb{B}$, then $N_{\mathcal{S}}=(V, E, w)$ does not carry more information in its weight function than its underlying digraph.

\footnotetext{
3 The definitions and notations in this section are those of [32] applied to matrices with entries and graphs with weights in semirings.
} 
The paths and cycles of a network $N_{\mathcal{S}}$ are those of its underlying digraph and for each path $p \in \Pi_{N}^{+}$its path prod$u c t$, or simply weight, is the lifting of the weight function to paths, $w: \Pi_{N}^{+} \rightarrow S, p \mapsto w(p)=\bigotimes_{l=1}^{k} w\left(i_{l-1}, i_{l}\right)$. For each set of paths $P \subseteq \Pi_{N}^{+}$its path sum, or weight, is the lifting of the weight function to sets of paths: $w: 2^{\Pi_{N}^{+}} \rightarrow S, P \mapsto w(P)=\sum_{p \in P} w(p)$, with $w(\varnothing)=\epsilon$. In radicable semirings, a special type of path weight is used for cycles: for $c \in C_{N}^{+}$, call its cycle mean the geometric mean of its weight, $\mu(c)={ }^{l(c)} \sqrt{w(c)}=w(c)^{\frac{1}{l(c)}}$. For a set of cycles $C \subseteq C_{N}^{+}$the aggregated cycle mean is $\mu_{\oplus}(C)=\sum_{c \in C} \mu(c)$.

\section{Example 5.}

1. In the Boolean semiring the aggregated cycle mean just describes whether there is any cycle in the digraph.

2. The maxplus semiring is radicable and selective and the aggregated cycle mean is called the maximal cycle mean, $\mu_{\max }(C)=\max _{c \in C} \frac{w(c)}{l(c)}$.

In a selective semiring, a cycle that attains the aggregated cycle mean is called a critical cycle of $N_{\mathcal{S}}$. Therefore the set of critical cycles is $C_{N}^{c}=\arg \sum_{c \in C(G)} \mu(c)$. The critical vertex set is the union of vertices in the critical cycles, and the critical sub-digraph the union of its critical cycles $G_{N}^{c}=\bigcup\left\{c \mid c \in C_{N}^{c}\right\}$ as graphs.

Given $A \in \mathcal{M}_{n}(\mathcal{S})$, the network (weighted digraph) induced by $A, N_{A}=\left(V_{A}, E_{A}, w_{A}\right)$, consists of a set of vertices $V_{A}=\bar{n}$, a set of arcs, $E_{A}=\left\{(i, j) \mid A_{i j} \neq \epsilon_{S}\right\}$, and a cost function $w_{A}: V_{A} \times V_{A} \rightarrow S,(i, j) \mapsto w_{A}(i, j)=a_{i j}$. This allows us to apply intuitively all notions from networks to matrices and vice versa, like the underlying graph $G_{A}=\left(V_{A}, E_{A}\right)$, the set of paths $\Pi_{A}^{+}(i, j)$ between nodes $i$ and $j$ or the set of normal $C_{A}^{+}(i)$ or elementary cycles $C_{A}^{e}(i)$ through node $i$. The following result transforms intuitions on matrix closures into intuitions about path weights in the associated network:

Proposition 2.10. Let $A \in \mathcal{M}_{n}(\mathcal{S})$ be a matrix over semiring $\mathcal{S}$. Then,

1. $A_{i j}^{k}=w_{A}\left(\Pi_{A}^{k}(i, j)\right), A_{i j}^{+k}=w_{A}\left(\Pi_{A}^{+k}(i, j)\right)$.

2. If $A^{+}$exists,

(a) $A_{i j}^{+}=w_{A}\left(\Pi_{A}^{+}(i, j)\right)$ and $A_{i i}^{+}=w_{A}\left(C_{A}^{+}(i)\right)$.

(b) If there is a non-null $\mu \in S$ and a vertex $i \in \bar{n}$ such that $w_{A}\left(C_{A}^{+}(i)\right) \otimes \mu \oplus \mu=w_{A}\left(C_{A}^{+}(i)\right) \otimes \mu$ then $A_{\cdot i}^{+} \otimes \mu$ is an eigenvector of A for $e$.

3. If $\mathcal{S}$ is entire and zerosumfree then $A_{i i}^{+}=\epsilon$ iff $C_{A}^{+}(i)=\varnothing$.

4. If $\mathcal{S}$ is complete and multiplicatively-cancellative and there is some $c \in C_{A}^{+}(i)$ with $w_{A}(c) \succ e$, then $A_{i i}^{+}=\top$. Furthermore, if $\top$ is strongly infinite, $A_{i j}^{+}=\top$ for vertices $j$ reachable from any vertex $i$ in such $c$.

5. If $\mathcal{S}$ is complete and idempotent and $w_{A}(c) \preccurlyeq e$ for all $c \in C_{A}^{+}(i)$, then $A_{i i}^{*}=e$.

6. If $\mathcal{S}$ is a complete selective multiplicatively-cancellative semiring and $C_{A}^{+}(i) \neq \varnothing$, then $A_{i i}^{*} \in\{e, \top\}$.

Proof. For claims 1 and 2 a consult [14, Chap. 4, Property 3.2.1].

For claim $2 \mathrm{~b}$ recall that $A^{+}$and $A^{*}$ only differ in their diagonals. If the condition is true, by claim $2 \mathrm{a}$ we get $A_{i i}^{+} \otimes \mu=\left(A_{i i}^{+} \oplus e\right) \otimes \mu$ whence by Theorem $2.4 A_{. i}^{+} \otimes \mu \in \mathcal{V}_{e}(A)$.

For 3, by claim 2a if $C_{A}^{+}(i)=\varnothing$ then $A_{i i}^{+}=w_{A}(\varnothing)=\perp$. Since $\mathcal{S}$ is entire and zerosumfree, no path sum may be null otherwise. Recall that all stars exist in complete semirings, so if $c \in C_{A}^{+}(i)$ then $c^{k} \in C_{A}^{+}(i)$-where $c^{k}$ is the concatenation of $k$ of these cycles-and $w_{A}\left(c^{k}\right)=w_{A}^{k}(c)$. Call $c^{+}=\lim _{n \mapsto \infty} \bigcup_{k=1}^{n} c^{k}$ whence $w_{A}\left(c^{+}\right)=$ $\lim _{n \mapsto \infty} \sum_{k=1}^{n} w_{A}\left(c^{k}\right)=w_{A}(c)^{+}$whence $A_{i i}^{+}=w_{A}\left(C_{i}^{+}\left(G_{A}\right)\right) \succcurlyeq w_{A}\left(c^{+}\right)=w_{A}(c)^{+}$. In the conditions of claim 4, if $w_{A}(c) \succ e$ by Proposition 2.2, claim 3, we have $w_{A}(c)^{+}=\top$, so $A_{i i}^{+}=\top$. For claim 5, since $\mathcal{S}$ is idempotent and complete, by Proposition 2.2, claim $4 \mathrm{~b}$, if $w_{A}(c) \preccurlyeq e$ then $w_{A}(c)^{*}=e$, whence $A_{i i}^{*}=e$. Claim 6 is a corollary of claims 3-5 and claim 5 of Proposition 2.2.

Note that the existence of the completion procedure in Example 2 guarantees the existence of many dioids in which the conditions for claim 4 hold, including all dioids with multiplicative group structure, such as $\overline{\mathbb{R}}_{\max ,+}$ or $\overline{\mathbb{R}}_{+, x}^{+}$, or even $\overline{\mathbb{N}}_{+, x}$. 
Finally, matrix scaling affects the induced network, but not the underlying graph:

Lemma 2.11. Let $A \in \mathcal{M}_{n}(\mathcal{S})$ be a matrix over semiring $\mathcal{S}$. If $\alpha \neq \epsilon \in S$ then:

1. $G_{\alpha \otimes A}=G_{A}$ and $w_{\alpha \otimes A}(p)=\alpha^{l(p)} \otimes w_{A}(p)$ for $p \in \Pi_{A}^{+}$.

2. If $\mathcal{S}$ is further radicable, then $\mu_{\alpha \otimes A}(c)=\alpha \otimes \mu_{A}(c)$ for all $c \in C_{A}^{+}$.

3. If $\mathcal{S}$ is further a radicable dioid, $G_{\alpha \otimes A}^{c}=G_{A}^{c}$. But if $\mathcal{S}$ has a strongly infinite element $\alpha=\top$ only $G_{\top \otimes A}^{c} \supseteq G_{A}^{c}$ holds.

Proof. Since $V_{A}=V_{\alpha \otimes A}$ and $E_{A}=E_{\alpha \otimes A}$ then $G_{A}=G_{\alpha \otimes A}$. If $p=\left(i_{0}, \ldots, i_{k}\right) \in \Pi_{A}^{+}$with $l(p)=k$ then we know $w_{\alpha \otimes A}(p)=\left(\alpha \otimes a_{i_{0} i_{1}}\right) \otimes \cdots \otimes\left(\alpha \otimes a_{i_{k-1} i_{k}}\right)=\alpha^{k} \otimes w_{A}(p)$. If $\mathcal{S}$ is radicable and $p$ is a cycle, $\mu_{\alpha \otimes A}(c)=\alpha \otimes \mu_{A}(c)$. Since product and order are compatible in a dioid, the critical character is maintained in $\alpha \otimes A$ in incomplete dioids. Of course $\top \otimes A$ makes all of its cycles critical so $G_{\top \otimes A}^{c}=C_{A}^{+} \supseteq G_{A}^{c}$.

\section{The spectra of irreducible matrices over completed idempotent dioids}

First we gather some very general results, mostly either of combinatorial in nature or holding in interesting classes of semirings (Section 3.1). We finally concentrate on irreducible matrices over complete semifield case (Section 3.2). We will use the notation of complete semirings throughout but caution that in generic semirings $\oplus, \otimes$ default to $\oplus, \otimes$.

\subsection{General results}

The proofs of Propositions 2.4 and 5.1 highlight the role of transitive closures. Inconveniently, the very stringent condition that $A^{*}$ exists deters practitioners from using it. Recall that a semiring $\mathcal{S}$ is complete, if for any index set $I$ including the empty set, and any $\left\{a_{i}\right\}_{i \in I} \subseteq \mathcal{S}$ the (possibly infinite) summations $\bigoplus_{i \in I} a_{i}$ are defined and the distributivity conditions: $\left(\bigoplus_{i \in I} a_{i}\right) \otimes c=\bigoplus_{i \in I}\left(a_{i} \otimes c\right)$ and $c \otimes\left(\bigoplus_{i \in I} a_{i}\right)=\bigoplus_{i \in I}\left(c \otimes a_{i}\right)$, are satisfied. Note that for $c=e$ the above demand that infinite sums have a result. Luckily, since completion in a semiring lifts to its induced matrix semirings, $A^{+}$exists for every $A \in S^{n \times n}$ as soon as $\mathcal{S}$ is complete. In such case, the top element $\top$, the supremum in the canonical order $\top \oplus a=\top$, is the sum of all the elements in the dioid $\top=\bigoplus_{a \in D} a$. By the semiring axioms, however, we have: $\top \otimes \epsilon=\epsilon$. Note that if $a \otimes b=\top$ then $a=\top$ or $b=\top$ or both.

As a partially-ordered set, $\mathrm{a} \vee$-semilattice is complete when the lowest upper bound operates on arbitrary subsets of $S$ and likewise for complete $\wedge$-semilattices. Lattices are complete when both their $\vee$ - and $\wedge$-semilattices are complete, hence they have both a top and a bottom. From a well-known order-theory theorem-a complete $\vee$-semilattice with bottom is also a complete lattice [33, Theorem 2.31, p. 47] — it is clear that complete idempotent semirings are already complete lattices, whence,

Corollary 3.1. Let $A \in \overline{\mathcal{K}}^{n \times n}$ be a matrix with entries in a commutative complete idempotent semiring. For all eigenvalues $\rho \in \mathrm{P}(A), \mathcal{V}_{\rho}(A)$ is a complete lattice.

Without loss of generality, from now on call $\top$ any strongly infinite element of $\mathcal{S}$. Corollary 3.1 highlights the novelty afforded by complete dioids and preludes the following crucial difference between spectra in completed and incomplete semirings:

Proposition 3.2 (Improper spectrum). Let $A \in \mathcal{M}_{n}(\overline{\mathcal{S}})$ be a matrix with entries in an entire zerosumfree semiring with strongly infinite element $\top \in \overline{\mathcal{S}}$. If $\rho \neq \epsilon$ and $v \in \mathcal{V}_{\rho}(A), v \neq \epsilon^{n}$ then,

1. $v \otimes \top \in \mathcal{V}_{\rho}(A) \cap \mathcal{V}_{\rho^{\prime}}(A)$, with $\rho^{\prime} \in \bar{S} \backslash\{\epsilon\}$.

2. $\mathrm{P}(A) \supseteq \bar{S} \backslash\{\epsilon\}$.

Proof. If $v \in \mathcal{V}_{\rho}(A), v \neq \epsilon^{n}$, by the associativity of $\otimes$ and since $\top$ is strongly infinite 


$$
\begin{aligned}
A \otimes(v \otimes T) & =(A \otimes v) \otimes T=(v \otimes \rho) \otimes T=v \otimes(\rho \otimes T)=v \otimes(T \otimes \rho) \\
& =v \otimes(T \otimes T)=v \otimes\left(T \otimes \rho^{\prime}\right)=(v \otimes T) \otimes \rho^{\prime},
\end{aligned}
$$

for $\rho^{\prime} \neq \rho \in \bar{D} \backslash\{\epsilon\}$, proving both claims.

This raises a terminological issue since we would like to distinguish between the proper eigenvalues, like those afforded by the spectral theory on matrices over incomplete dioids, and the induced or improper eigenvalues in Proposition 3.2. Since the situation is brought about by $v \otimes T$ having only non-finite coordinates, call the support of $a$ vector the set of indices of $v$ whose coordinates are non-null, $\operatorname{supp}(v)=\left\{k \in \overline{\mathbf{n}} \mid v_{k} \neq \epsilon\right\}$. We say that $v$ has full support if all of its coordinates are non-null, otherwise we say that it has partial support. For the case of complete semirings, call the saturated support of an eigenvector the set of indices of $v$ whose coordinates are the infinite, sat-supp $(v)=\left\{k \in \overline{\mathbf{n}} \mid v_{k}=T\right\}$. The rest of the support is the finite support, fin-supp $(v)=\left\{k \in \overline{\mathbf{n}} \mid \epsilon \neq v_{k} \neq \top\right\}$.

We propose to call an eigenvalue proper when it has at least one eigenvector with finite coordinates, otherwise it is improper. The set of proper (left) eigenvalues is the proper (left) spectrum,

$$
\begin{aligned}
& \mathrm{P}^{\mathrm{P}}(A)=\left\{\rho \in \mathrm{P}(A) \mid \exists v \in \mathcal{V}_{\rho}(A) \text { fin-supp }(v) \neq \varnothing\right\} \\
& \left(\Lambda^{\mathrm{P}}(A)=\left\{\lambda \in \Lambda(A) \mid \exists u \in \mathcal{U}_{\lambda}(A), \text { fin-supp }(u) \neq \varnothing\right\}\right),
\end{aligned}
$$

so the improper (left) spectrum is $\mathrm{P}(A) \backslash \mathrm{P}^{\mathrm{P}}(A)$ (respectively, $\Lambda(A) \backslash \Lambda^{\mathrm{P}}(A)$ ).

\subsubsection{General results on supports}

We use the following shorthand for proofs: $J_{\top}=\operatorname{sat}-\operatorname{supp}(v), J_{\mathrm{F}}=\operatorname{fin}-\operatorname{supp}(v)$ and $J_{\epsilon}=(\operatorname{supp}(v))^{\mathrm{C}}$ for the complement of the support, with $n_{1}=\left|J_{\top}\right|, n_{2}=\left|J_{\mathrm{F}}\right|, n_{3}=\left|J_{\epsilon}\right|$ and $v_{\mathrm{F}}=v_{J_{\mathrm{F}}}$ and $A_{x y}=A_{J_{x} J_{y}}$. From the permutation induced by $\overline{\mathbf{n}}=J_{\top} \cup J_{\mathrm{F}} \cup J_{\epsilon}$,

$$
\begin{aligned}
& {\left[\begin{array}{ccc}
A_{\mathrm{TT}} & A_{\mathrm{TF}} & A_{\top \epsilon} \\
A_{\mathrm{FT}} & A_{\mathrm{FF}} & A_{\mathrm{F} \epsilon} \\
A_{\epsilon \mathrm{T}} & A_{\epsilon \mathrm{F}} & A_{\epsilon \epsilon}
\end{array}\right] \otimes\left[\begin{array}{c}
T^{n_{1}} \\
v_{\mathrm{F}} \\
\epsilon^{n_{3}}
\end{array}\right]=\left[\begin{array}{c}
T^{n_{1}} \\
v_{\mathrm{F}} \\
\epsilon^{n_{3}}
\end{array}\right] \otimes \rho,} \\
& A_{\top \mathrm{T}} \otimes T^{n_{1}} \oplus A_{\top \mathrm{F}} \otimes v_{\mathrm{F}}=\top^{n_{1}} \otimes \rho \\
& A_{\mathrm{FT}} \otimes T^{n_{1}} \oplus A_{\mathrm{FF}} \otimes v_{\mathrm{F}}=v_{\mathrm{F}} \otimes \rho \\
& A_{\epsilon \mathrm{T}} \otimes T^{n_{1}} \oplus A_{\epsilon \mathrm{F}} \otimes v_{\mathrm{F}}=\epsilon^{n_{3}} .
\end{aligned}
$$

Lemma 3.3. Let $A \in \mathcal{M}_{n}(\mathcal{S})$ over an entire zerosumfree semiring with $\rho \in \mathrm{P}(A), v \in \mathcal{V}_{\rho}(A)$. Then:

1. If $v$ has partial support, $A$ is reducible.

2. If $i \notin \operatorname{supp}(v)$, then for all $j \in \operatorname{supp}(v), a_{i j}=\epsilon$.

3. The eigenequations become:

$$
\forall i \in \operatorname{supp}(v), \quad \bigoplus_{k \in \operatorname{supp}(v)} a_{i k} \otimes v_{k}=v_{i} \otimes \rho
$$

4. If $\rho=\epsilon$, then for all $i, j \in \operatorname{supp}(v), a_{i j}=\epsilon$.

Proof. If $v$ has partial support, (8c) entails that $A_{\epsilon \mathrm{F}}=\epsilon^{n_{3} \times n_{2}}$ so by definition $A$ is reducible. Claim 2 is just another way to state the preceding. Claim 3, then, is (8a) and (8b) put together, and claim 4 follows from (8a) and (8b).

As the contrapositive of Lemma 3.3, claim 1 we generalize the well known:

Corollary 3.4. (See [34, Lemma 5.5.4, slightly generalized].) Let $A \in \mathcal{M}_{n}(\mathcal{S})$ be an irreducible matrix over an entire zerosumfree semiring. Then, if $v \in \mathcal{V}_{\rho}(A)$ with $v \neq \epsilon^{n}$, it is fully-supported, $\operatorname{supp}(v)=\overline{\mathbf{n}}$. 
In contrast, saturated supports may arise even for finite eigenvalues:

Lemma 3.5. Let $A \in \mathcal{M}_{n}(\mathcal{S})$ over an entire zerosumfree semiring with a strongly infinite element $\top$ with $\rho \neq \epsilon$ and $v \in \mathcal{V}_{\rho}(A)$. Then:

1. If $a_{i j}=\top$ and $j \in \operatorname{supp}(v)$ then $i \in \operatorname{sat}-\operatorname{supp}(v)$.

2. If $a_{i j} \neq \epsilon$ and $j \in \operatorname{sat}-\operatorname{supp}(v)$ then $i \in \operatorname{sat}-\operatorname{supp}(v)$.

3. If $\rho=\top$, for all $i \in \operatorname{fin}-\operatorname{supp}(v)$ either there exists $k \in \operatorname{fin}-\operatorname{supp}(v), a_{i k}=\top$, or there exists $j \in \operatorname{sat}-\operatorname{supp}(v)$, $a_{i j} \neq \epsilon$, or both.

4. If $\epsilon \neq \rho \neq \top$ then:

(a) For all $i \in \operatorname{fin}-\operatorname{supp}(v)$, there exists $k \in \operatorname{fin}-\operatorname{supp}(v), a_{i k} \neq \epsilon$, and for all $j \in \operatorname{sat}-\operatorname{supp}(v), a_{i j}=\epsilon$. That $i s, A_{\mathrm{FF}}$ has no null rows but $A_{\mathrm{FT}}$ is null.

(b) If $v$ has full support but partial finite support, A is reducible.

Proof. Claims 1, 2, 3 and $4 \mathrm{a}$ are read from (8a) and (8b). For claim $4 \mathrm{~b}$, if $i \in$ fin-supp $(v)$ then since $\rho$ is finite, from (8b) follows that $A_{\mathrm{FT}}=\epsilon^{n_{2} \times n_{1}}$.

\subsubsection{General results on the null eigenvalue and its eigenspace}

Call $e_{i}=I_{i}$ the $i$-th column of $I$, the unit in the semiring of matrices $\mathcal{M}_{n}(S)-e_{i}$ is a vector whose coordinates are zero except for $e_{i}(i)=e$-and note that $A \otimes e_{i}=A_{. i}$.

Lemma 3.6. (See [34, Lemmas 5.5.1 and 5.5.2, generalized].) Let $A \in \mathcal{M}_{n}(\mathcal{S})$ over a semiring. Then:

1. If the $i$-th column of $A$ is zero, then $e_{i} \in \mathcal{V}_{\epsilon}(A)$, whence $\epsilon \in \mathrm{P}^{\mathrm{P}}(A)$.

2. Further, if $\mathcal{S}$ is entire, then $G_{A}$ has no cycles if and only if $\epsilon$ is the unique eigenvalue of $A$.

3. Further, if $\mathcal{S}$ is entire and zerosumfree and $\epsilon \in \mathrm{P}(A)$, then $A$ has at least one zero column.

Proof. Suppose column $i \in \overline{\mathbf{n}}$ is zero $A_{\cdot i}=\epsilon^{n \times 1}$. Then $e_{i}$ is an eigenvector of $A$ for $\epsilon$ since $A \otimes e_{i}=A_{. i}=e_{i} \otimes \epsilon$. For claim 2, if $C_{A}^{+}=\varnothing$ without loss of generality we suppose cycle-free $A$ in Upper Frobenius Normal Form as in (9),

$$
A=\left[\begin{array}{ccccc}
\epsilon & a_{12} & \ldots & a_{1(n-1)} & a_{1 n} \\
\epsilon & \epsilon & \ldots & a_{2(n-1)} & a_{2 n} \\
\vdots & \vdots & \ddots & \vdots & \vdots \\
\epsilon & \epsilon & \ldots & \epsilon & a_{(n-1) n} \\
\epsilon & \epsilon & \ldots & \epsilon & \epsilon
\end{array}\right] .
$$

Since $A_{.1}=\epsilon^{n}, \epsilon \in \mathrm{P}^{\mathrm{P}}(A)$. Conversely, suppose there exists a non-null eigenvector $v \in \mathcal{V}_{\rho}(A)$ of $A$ for $\rho \neq \epsilon$. For the last row of the eigenequations of the matrix in (9) we have $\epsilon=v_{n} \otimes \rho$ and since $\mathcal{S}$ is zero-divisor free and $\rho \neq \epsilon$ by hypothesis, we have $v_{n}=\epsilon$. For the last but one row we have $a_{n-1 n} \otimes v_{n}=v_{n-1} \otimes \rho$ with $v_{n}=\epsilon$ so $v_{n-1}=\epsilon$. Proceeding this way we conclude that $v=\epsilon^{n \times 1}$, a contradiction.

Finally, let $v \in \mathcal{V}_{\epsilon}(A)$. From Lemma 3.3, claim 2 we know that $a_{i j}=\epsilon$ for $i \notin \operatorname{supp}(v), j \in \operatorname{supp}(v)$. Since $\rho=\epsilon$ we even know that $a_{i j}=\epsilon$ for $i, j \in \operatorname{supp}(v)$. Hence if there is some $j \in \operatorname{supp}(v)$ then for all $i \in \overline{\mathbf{n}}, a_{i j}=\epsilon$.

Call the columns of $I$ selected by the set of zero columns of $A$, the fundamental eigenvectors of $A$ for $\epsilon, \operatorname{FEV}_{\epsilon}(A)=$ $\left\{I_{\cdot i} \mid A_{\cdot i}=\epsilon^{n}\right\}$. The name is justified by,

Proposition 3.7. Let $A \in \mathcal{M}_{n}(\mathcal{S})$ over an entire zerosumfree semiring. The null eigenspace is generated by the fundamental eigenvectors of $A$ for $\epsilon$.

$$
\mathcal{V}_{\epsilon}(A)=\left\langle\operatorname{FEV}_{\epsilon}(A)\right\rangle_{\mathcal{S}}
$$

Proof. Call $\overline{z c}=\left\{i \in \overline{\mathbf{n}} \mid A_{\cdot i}=\epsilon^{n}\right\}$. Surely $\mathcal{V}_{\epsilon}(A) \supseteq\left\langle I_{\overline{z c}}\right\rangle_{\mathcal{S}}$, so suppose $v \in \mathcal{V}_{\epsilon}(A)$ with $v_{j} \neq \epsilon$ and $j \notin \overline{z c}$. Then $A . j \neq \epsilon^{n \times 1}$ so, for instance, $a_{i j} \neq \epsilon$. Then $(A \otimes v)_{i} \neq \epsilon=v \otimes \epsilon$ whence $v$ is not an eigenvector of $A$ for $\epsilon$, a contradiction. 
As a side note, this completely describes the eigenspace of the null eigenspace for the information algebras of Fig. 1, being quite different to the null eigenspace for fields.

\subsubsection{General results on non-null eigenvalues and their eigenspaces}

By definition, $\mathrm{P}^{\mathrm{P}}(A) \subseteq \mathrm{P}(A)$. Furthermore,

Corollary 3.8. Let $A \in \mathcal{M}_{n}(\overline{\mathcal{D}})$ be a matrix over an entire zerosumfree semiring with strongly infinite element and $\epsilon \neq \rho \in \mathrm{P}^{\mathrm{P}}(A)$. Then

1. if $A$ has no zero columns, $\mathrm{P}(A)=\bar{D} \backslash\{\perp\}$,

2. if A has no zero rows, $\Lambda(A)=\bar{D} \backslash\{\perp\}$.

Proof. By Proposition 3.2, claim 2 and Lemma 3.6, claim 3, since $A$ has no zero columns, $\mathrm{P}(A)=\mathcal{D} \backslash\{\perp$. Since $\Lambda(A)=\mathrm{P}\left(A^{\mathrm{T}}\right)$, claim 2 follows.

Once the improper spectrum characterized, we would like to start elucidating the relation between $\mathrm{P}^{\mathrm{P}}(A)$ and the cycle structure of $A$, as suggested by Lemma 3.6. So consider each cycle $c \in C_{A}^{+}$a subgraph $c=\left(V_{c}, E_{c}\right)$ of $G_{A}$, and for each eigenvector $v \in \mathcal{V}_{\rho}(A)$ define the sets of (possibly partially):

- unsupported cycles, $C^{c}(v)=\left\{c \in C_{A}^{+} \mid V_{c} \cap \operatorname{supp}^{c}(v) \neq \varnothing\right\}$,

- supported cycles, $C(v)=\left\{c \in C_{A}^{+} \mid V_{c} \cap \operatorname{supp}(v) \neq \varnothing\right\}$,

- finitely-supported cycles, $C^{\mathrm{FIN}}(v)=\left\{c \in C_{A}^{+} \mid V_{c} \cap\right.$ fin-supp $\left.(v) \neq \varnothing\right\}$,

- saturatedly-supported cycles, $C^{\mathrm{SAT}}(v)=\left\{c \in C_{A}^{+} \mid V_{c} \cap\right.$ sat-supp $\left.(v) \neq \varnothing\right\}$,

and we say that $c \in C_{A}^{+}$is in the support of vector $v$ if $c \in C(v)$, and so on. Note that $C(v) \cup C^{c}(v)=C_{A}^{+}$and $C^{\mathrm{FIN}}(v) \cup C^{\mathrm{SAT}}(v)=C(v)$. Lemma 3.9 states that any eigenvector for $\rho \neq \epsilon$ actually partitions the set of cycles of a matrix:

Lemma 3.9. Let $A \in \mathcal{M}_{n}(\mathcal{S})$ be a matrix over an entire zerosumfree semiring with $v \in \mathcal{V}_{\rho}(A)$ and $\rho \neq \epsilon$. Then:

1. A has at least a cycle, $C_{A}^{+} \neq \varnothing$.

2. Cycles are either totally supported or unsupported by $v, C(v) \cap C^{c}(v)=\varnothing$.

3. If $S$ is further an entire dioid with strongly infinite element $T$, then:

(a) If $\perp \prec \rho \prec T$ then those cycles supported by $v$ are totally supported whether finitely or saturatedly, $C^{\mathrm{FIN}}(v) \cap$ $C \mathrm{SAT}(v)=\varnothing$.

(b) If $C^{\mathrm{FIN}}(v) \cap C^{\mathrm{SAT}}(v) \neq \varnothing$ then $\rho=\mathrm{T}$.

Proof. The first claim is the contrapositive of Lemma 3.6, claim 2. For claim 2, let $1 \preccurlyeq k \preccurlyeq K=\left|V_{c}\right|$ index the vertices of $c$ (as dictated by $E_{c}$ ). For each $i_{k} \in V_{c}$, we have from (7) for node $i_{k-1}$ that: $\sum_{j \in \operatorname{supp}(v)} a_{i_{k-1} j} \otimes v_{j}=v_{i_{k-1}} \otimes \rho$, but if $i_{k} \in \operatorname{supp}(v)$, then $v_{i_{k}} \neq \epsilon$, since it belongs to the cycle $a_{i_{k-1} i_{k}} \neq \epsilon$ and $\rho \neq \epsilon$, so we must have $v_{i_{k-1}} \neq \epsilon$ that is $i_{k-1} \in \operatorname{supp}(v)$. The preceding reasoning serves as induction case, so if we suppose that $i_{K} \in \operatorname{supp}(v)$, this means $V_{c} \subseteq \operatorname{supp}(v)$. As any vertex in the cycle can be chosen for $i_{K}$, this applies to all $i_{k}$.

For claim 3 a from Lemma 3.5, claim 4, we know that $A_{\mathrm{FT}}$ is zero, hence no cycle may span both finite and saturated supports. Claim $3 \mathrm{~b}$ is just its contrapositive.

The following lemma highlights the importance of cycle weights in dioids:

Lemma 3.10. (Compare to [14, Chap. 6, Th. 2].) Let $A \in \mathcal{M}_{n}(\mathcal{D})$ be a matrix over a commutative dioid with $\rho \neq \perp$ and $v \in \mathcal{V}_{\rho}(A)$. Then, 
1. For any cycle $c \in C(v)$ of length $l(c)$,

$$
w_{A}(c) \otimes v_{i} \preccurlyeq v_{i} \otimes \rho^{l(c)}, \quad \text { for } i \in V_{c} .
$$

2. If $\mathcal{D}$ is further selective, there is a cycle $c^{\prime} \in C(v)$ of length $l\left(c^{\prime}\right)$ such that

$$
w_{A}\left(c^{\prime}\right) \otimes v_{i}=v_{i} \otimes \rho^{l\left(c^{\prime}\right)}, \quad \text { for } i \in V_{c^{\prime}} .
$$

Proof. Consider cycle $c=\left(i_{1}, i_{2}, i_{3}, \ldots, i_{k}\right)$ of length $l(c)=k$ in the support of $v$. From the eigenequations (8a)-(8c), for the vertices in the cycle we have:

$$
\begin{aligned}
& a_{i_{1} i_{2}} \otimes v_{i_{2}} \preccurlyeq v_{i_{1}} \otimes \rho \\
& a_{i_{2} i_{3}} \otimes v_{i_{3}} \preccurlyeq v_{i_{2}} \otimes \rho \\
& a_{i_{k} i_{1}} \otimes v_{i_{1}} \preccurlyeq v_{i_{k}} \otimes \rho .
\end{aligned}
$$

Multiplying (13b) by $a_{i_{1} i_{2}}$ and then introducing (13a), by the compatibility of the product and the order, we get $a_{i_{1} i_{2}} \otimes a_{i_{2} i_{3}} \otimes v_{i_{3}} \preccurlyeq v_{i_{1}} \otimes \rho^{2}$. By iterating on the other inequalities we get claim 1 as $a_{i_{1} i_{2}} \otimes \cdots \otimes a_{i_{k} i_{1}} \otimes v_{i_{1}} \preccurlyeq v_{i_{1}} \otimes \rho^{k}$.

When $\mathcal{D}^{\circ}$ is selective, for each $i \in \operatorname{supp}(v)$ the sum in the eigenequations is attained at a particular index $\dot{\varphi}(i) \in$ $\operatorname{supp}(v)$ where $a_{i \varphi(i)} \otimes v_{\varphi(i)}=v_{i} \otimes \rho$. Gather the edges $E_{H}=\left\{(i, \varphi(i)) \mid a_{i \varphi(i)} \otimes v_{\varphi(i)}=v_{i} \otimes \rho, i \in \operatorname{supp}(v)\right\}$ and consider the partial graph $H=\left(\operatorname{supp}(v), E_{H}\right) \subseteq G_{A}$ : it has $|\operatorname{supp}(v)|$ vertices and arcs hence its cyclomatic number equals its connectivity number, $K$. Let $H=\bigcup_{k=1}^{K} H_{k}$, where $H_{k}$ is a connected component of $H$. Since every vertex has out-degree 1, each connected component contains a single circuit $\left\{c^{k}\right\}_{k=1}^{K}$. On any such circuit $c^{k}$, supported by construction, (13a)-(13c) hold with equality so claim 2 follows.

Notice that if $\top$ is a strongly infinite element of $\mathcal{D}$, when $v_{i}=\top$ (11) and (12) are not very informative; we need to work in finite supports, hence with proper eigenvalues:

Lemma 3.11 (Witness cycles). Let $A \in \mathcal{M}_{n}(\overline{\mathcal{K}})$ over a completed idempotent semifield. Let $\rho \in \mathrm{P}^{\mathrm{P}}(A)$ such that $\perp \prec \rho$ and $v \in \mathcal{V}_{\rho}(A)$. Then,

1. For any cycle $c \in C(v)$ of length $l(c)$,

(a) If $w_{A}(c) \succ \rho^{l(c)}$ then $V_{c} \subseteq \operatorname{sat}-\operatorname{supp}(v)$.

(b) If $V_{c} \cap \operatorname{fin}-\operatorname{supp}(v) \neq \varnothing$, then $w_{A}(c) \preccurlyeq \rho^{l(c)}$.

2. If $\overline{\mathcal{K}}$ is further selective and fin-supp $(v) \neq \varnothing$ there is a cycle $c^{\prime} \in C^{\mathrm{FIN}}(v)$ of length $l\left(c^{\prime}\right)$ such that $w_{A}\left(c^{\prime}\right)=\rho^{l\left(c^{\prime}\right)}$, and we call it $a$ witness cycle for $\rho$.

3. If $\overline{\mathcal{K}}$ is further selective and radicable, then all such witness cycles are critical among the cycles (partially) finitely supported by $v$, and $\rho$ is their shared mean,

$$
\mu_{A}\left(c^{\prime}\right)=\sqrt[l\left(c^{\prime}\right)]{w_{A}\left(c^{\prime}\right)}=\rho \quad \Leftrightarrow \quad c^{\prime} \in \arg \mu_{A}^{\oplus}\left(C^{\mathrm{FIN}}(v)\right) .
$$

Proof. For claim 1 a suppose $w_{A}(c) \succ \rho^{l(c)}$. If $i \in V_{c} \cap$ fin-supp $(v) \neq \varnothing$ since $\overline{\mathcal{K}}$ is a semifield we may multiply both sides of Lemma 3.10, claim 1, by $v_{i}^{-1}$ to obtain a contradiction. Claim $1 \mathrm{~b}$ is just the contrapositive, but worth stating.

Note that under the conditions of claim $1 \overline{\mathcal{K}}$ is already totally-ordered by the remarks after Proposition 2.3. In the proof of Lemma 3.10, claim 2, consider the cycles $\left\{c^{k}\right\}_{k=1}^{K}$. Since $v$ is finitely supported, for one of those cycles $c^{\prime}$, at least there is $\perp \prec v_{i} \prec \top$, hence its inverse exists and claim 2 follows.

The equality of the eigenvalue and the cycle mean follows from claims 1 and 2 and the properties of radicable semirings. The characterization of the cycles follows from claim 2 when we restrict ourselves to (partially) supported cycles.

Recall that when $\rho$ is the maximal cycle mean $\rho=\mu_{\oplus}(A)$ its witness cycles are customarily called critical. The top eigenvalue may be proper if it is a cycle mean: 
Corollary 3.12. Let $A \in \mathcal{M}_{n}(\bar{K})$ be a matrix with entries in a completed selective semifield. If $\top \in \mathrm{P}^{\mathrm{P}}(A)$ then there is a cycle $c \in C_{A}^{+}$such that $a_{i j}=\top$ for $(i, j) \in E_{c}$.

Proof. By Lemma 3.11, claim 2, we surely have a $c$ such that $w_{A}(c)=T^{l(c)}$, whence $w_{A}(c)=\top$. Since $l(c) \leq n$ this must mean some of the edges in the cycle, say $(i, j)$, have a weight of $a_{i j}=\mathrm{T}$.

Corollary 3.13. Let $A \in \mathcal{M}_{n}(\overline{\mathcal{K}})$ be a matrix over a complete selective radicable semifield. Only cycle means may be non-null proper eigenvalues,

$$
\mathrm{P}^{\mathrm{P}}(A) \backslash\{\perp\} \subseteq\left\{\mu_{\oplus}(c) \mid c \in C_{A}^{+}\right\} .
$$

Proof. This follows from claims 2 and 3 of Lemma 3.11, and Corollary 3.12.

\subsubsection{On a finite eigenvalue and its eigenspace}

If $\overline{\mathcal{K}}$ is a completed naturally-ordered semifield for $\perp<\rho<T$, define a normalized matrix $\tilde{A}^{\rho}=A / \rho=\rho^{-1} \dot{\otimes} A$.

By Lemma 2.11, $G_{\tilde{A}^{\rho}}=G_{A}$, and in particular $G_{\tilde{A}^{\rho}}^{c}=G_{A}^{c}$ and $\mu_{\tilde{A}^{\rho}}(c)=\rho^{-1} \dot{\otimes} \mu_{A}(c)$. If there exists a cycle with $\mu_{A}(c)=\rho$ then $\mu_{\tilde{A}^{\rho}}(c)=e$.

Lemma 3.14. Let $A \in \mathcal{M}_{n}(\overline{\mathcal{K}})$ over a completed semifield and $\perp<\rho<T$. Then:

1. $v$ is an eigenvector of $A$ for $\rho$ iff it is an eigenvector of $\tilde{A}^{\rho}$ for $e$.

2. $\rho$ is a proper eigenvalue of $A$ iff $e$ is a proper eigenvalue of $\tilde{A}^{\rho}$.

Proof. For finite values of $\rho, \rho^{-1} \dot{\otimes} A=\rho^{-1} \otimes A=\rho^{-1} \otimes A$, hence we may write $v \in \mathcal{V}_{\rho}(A) \Leftrightarrow A \otimes v=v \otimes \rho \Leftrightarrow$ $\rho^{-1} \otimes(A \otimes v)=\left(\rho^{-1} \otimes A\right) \otimes v=v \Leftrightarrow v \in \mathcal{V}_{e}\left(\tilde{A}^{\rho}\right)$, which also proves claim 2, as soon as $v$ has non-empty finite support.

Hence, for finite eigenvalues it is enough to work with $B=\tilde{A}^{\rho}$. Locating candidate eigenvectors for the unit eigenvalue is easy:

Proposition 3.15. Let $B \in \mathcal{M}_{n}(\overline{\mathcal{D}})$ over a complete selective multiplicatively cancellative dioid For $i \in \bar{n}$, the following are equivalent:

1. $B_{. i}^{+} \otimes \mu \in \mathcal{V}_{e}(B)$ with $\mu \notin\{\perp, \top\}$.

2. $B_{i i}^{+}=e$ or $B_{i i}^{+}=\mathrm{T}$.

3. Either there exists $c \in C_{B}^{+}(i)$ such that $w_{B}(c)>e$, or for all cycles passing through $i, w_{B}\left(c^{\prime}\right) \leq e$ with some $w_{B}(c)=e$.

Proof. Note that since $\overline{\mathcal{D}}$ is selective and entire it is totally-ordered. $(1 \Rightarrow 2)$ By Theorem 2.4 the condition equals $B_{i i}^{*} \otimes \mu=B_{i i}^{+} \otimes \mu$. Since $\overline{\mathcal{D}}$ is complete all stars exist and since it is multiplicatively cancellative for $\mu \notin\{\top, \perp\}, B_{i i}^{*}=B_{i i}^{+}$, whence from Proposition 2.10, claim 6, $B_{i i}^{+}=B_{i i}^{*}=\{e, \top\} .(2 \Rightarrow 3)$ By Proposition 2.10, claim 2a, $B_{i i}^{+}=\sum_{c \in C_{B}^{*}} w_{B}(c)^{+}$, but since $\overline{\mathcal{D}}$ is selective and totally-ordered Proposition 2.2, claim 5, describes the choices exhaustively: for $B_{i i}^{+}=\top$ we must have $w_{B}(c) \succ e$ for some $c \in C_{B}^{+}(i)$, and if $B_{i i}^{+}=e$ for every cycle $w_{B}\left(c^{\prime}\right) \preccurlyeq e$. But since $\overline{\mathcal{D}}$ is selective, in particular for some $c$ we must have $w_{B}(c)=e$. $(3 \Rightarrow 1)$ If $e \prec w_{A}(c)$ then $B_{i i}^{+}=B_{i i}^{*}=\top$, and if $w_{A}\left(c^{\prime}\right) \preccurlyeq e$ with some $w_{A}(c)=e$ then $B_{i i}^{+}=B_{i i}^{*}=e$, whence $B_{i i}^{+}=B_{i i}^{*}=\{e, \top\}$ so $B_{. i}^{+}=B_{. i}^{*}$ whence $B_{\cdot i}^{+} \otimes \mu \in \mathcal{V}_{e}(B)$ for all $\mu$.

For (incomplete) idempotent semifields, the way $\mathcal{V}_{e}(B)$ is generated is well understood [14, Ch. 6, Lemma 3.1, Theorem 2 and Corollary 3.2]. For complete commutative dioids, the following proposition allows us to detect the set 
of critical nodes $\bar{n}_{B}^{e}$ that index into the columns of the transitive closure matrix $B^{+}$to later extract the fundamental eigenvectors for eigenvalue $e$ :

Proposition 3.16. Let $B \in \mathcal{M}_{n}(\mathcal{D})$ be a matrix with entries in a complete commutative dioid $\mathcal{D}$ such that $e \in \mathrm{P}(B)$. Then:

1. $\mathcal{V}_{e}(B)$ is generated by the columns of $B^{+}$.

2. If $\mathcal{D}$ is further selective, then $\mathcal{V}_{e}(B)$ is generated by the subset of columns of $B^{+}$selected by the nodes in witness cycles, $\bar{n}_{B}^{e}$.

Proof. For claim 1, for every $v \in \mathcal{V}_{e}(B)$ we have $B \otimes v=v$. This is the initial step to induce that $B^{k} \otimes v=v$. Adding for $k \rightarrow \infty$ we have $v=B^{+} \otimes v$-since $B^{+}$always exists if $\mathcal{D}$ is complete—so the coordinates of an eigenvector are the coefficients to generate it from $B^{+}$.

For claim 2, by applying Lemma 3.10, claim 2, with $\rho:=e$ we get for all $i_{k} \in V_{c^{k}}, w_{B}\left(c^{k}\right) \otimes v_{i_{k}}=v_{i_{k}}$. Since $\mathcal{D}$ is idempotent $v_{i} \oplus v_{i}=v_{i}$ whence $w_{B}\left(c^{k}\right) \otimes v_{i_{k}} \oplus v_{i_{k}}=w_{A}\left(c^{k}\right) \otimes v_{i_{k}}$ so for all $i_{k}$ we have $\dot{B}_{i_{k}}^{+} \stackrel{\otimes}{\otimes} v_{i_{k}} \in \mathcal{V}_{e}(B)$ by Corollary 3.15 .

These are the only generators since, for each component $k$ in the proof of Lemma 3.10, let $c_{j i}$ be the single path from any non-cycle vertex $j$ to any vertex $i$ in the cycle. Since this path is of length $r \geq 1$ with $B_{j i}^{r}=w_{B}\left(c_{j i}\right)$, we have $w_{B}\left(c_{j i}\right) \otimes v_{i}=v_{j}$. As $\mathcal{D}$ is selective (hence idempotent), we have $B^{+} \oplus B^{*} \otimes B^{r}=B^{+}$, whence $B_{. i}^{+} \oplus$ $B_{\cdot j}^{*} \otimes w_{B}\left(c_{j i}\right)=\dot{B}_{\cdot i}^{+}$and then $B_{\cdot i}^{+} \otimes v_{i} \oplus B_{. j}^{+} \otimes v_{j}=B_{\cdot i}^{+} \otimes v_{i}$. That is, column $j$ is absorbed by column $i$ in $v=$ $B^{+} \otimes v$, so we need only retain those columns of $B^{+}$indexed by the nodes in the cycle in each component $\bar{n}_{B}^{e}\left(c^{k}\right)=$ $V_{c^{k}}$, whence $v=B_{\cdot \bar{n}_{B}^{e}}^{+} \otimes v_{\bar{n}_{B}^{e}}$ with $\bar{n}_{B}^{e}=\bigcup_{k=1}^{K} \bar{n}_{B}^{e}\left(c^{k}\right) \subseteq \operatorname{supp}(v)$.

\subsection{The spectra of irreducible matrices over completed idempotent semifields}

We describe the eigenspaces of improper eigenvalues straightforwardly:

Proposition 3.17 (Saturated eigenspace). Let $A \in \mathcal{M}_{n}(\overline{\mathcal{D}})$ be an irreducible matrix over a commutative dioid with a strongly infinite element. Then

1. if $\rho \in \mathrm{P}(A)$, then $\mathcal{V}_{\rho}(A) \supseteq\left\{\perp^{n}, \top^{n}\right\}$,

2. if $\rho \in \mathrm{P}(A) \backslash \mathrm{P}^{\mathrm{P}}(A)$, then $\mathcal{V}_{\rho}(A)=\left\{\perp^{n}, \top^{n}\right\}$.

Proof. By Corollary 2.8, $A \otimes T^{n}=T^{n}$ so $T^{n} \in \mathcal{V}_{e}(A)$. By claim 1 of Proposition 3.2, claim 1 follows. For improper eigenvalues these are the only possible eigenvectors.

Notice that all $\rho \in \mathrm{P}(A) \backslash \mathrm{P}^{\mathrm{P}}(A)$ have the same (non-trivial) eigenspace and that eigenspaces have non-unitary intersections, $\mathcal{V}_{\rho}(A) \cap \mathcal{V}_{\rho^{\prime}}(A)=\left\{\perp^{n}, \top^{n}\right\}$ for $\rho, \rho^{\prime} \in \mathrm{P}(A)$. In fact, since claim 1 of Proposition 3.17 asserts that it is embedded in any other eigenspace, we call this intersection the saturated eigenspace, $\mathcal{V}^{\top}(A)=\mathcal{V}_{\rho}(A)$ when $\rho \in \mathrm{P}(A) \backslash \mathrm{P}^{\mathrm{P}}(A)$. As a lattice, it is clearly boolean $\mathcal{V}^{\top}(A) \cong 2$. It would be tempting to discard improper eigenvalues as trivial-much as those eigenvalues with a single null eigenvalue are disregarded in incomplete semirings—but this will not do for reducible matrices [15].

On the other hand, detecting a proper top eigenvalue $\mu_{\oplus}(A)=\top$ is easy.

Proposition 3.18. Let $A \in \mathcal{M}_{n}(\bar{D})$ be an irreducible matrix with entries in a completed commutative selective dioid. Then $\top \in \mathrm{P}^{\mathrm{P}}(A)$ if and only if there is a cycle $c \in C_{A}^{+}$such that $a_{i j}=\top$ for $(i, j) \in E_{c}$. 
Proof. Corollary 3.12 accounts for the first implication. Now, let $e_{j}^{-1}$ be a vector such that $\left(e_{j}^{-1}\right)_{k}=e$ if $j=k$ and $\left(e_{j}^{-1}\right)_{k}=\top$ otherwise. When an $a_{i j}=\top, e_{j}^{-1}$ is an eigenvector of $\mathrm{T}$, since for every $k$ in the saturated support (8a) holds while for the finitely-supported $\left(e_{j}^{-1}\right)_{j}=e$ we have $a_{i j} \otimes e \oplus \ldots=\mathrm{T}$, hence $\mathrm{T}$ is proper.

For irreducible matrices over semifields we can strengthen Proposition 3.16:

Proposition 3.19. Let $B \in \mathcal{M}_{n}(\overline{\mathcal{K}})$ be an irreducible matrix over a complete selective semifield. The following are equivalent:

1. $e \in \mathrm{P}^{\mathrm{P}}(B)$.

2. $B_{i i}^{+}=e$ for $i \in \overline{n_{B}^{e}}$.

3. $\mathcal{V}_{e}(B)$ is the semimodule generated by those columns of $B^{+}$with $B_{i i}^{+}=e$.

Proof. $(1 \Rightarrow 2)$ Since $e \in \mathrm{P}^{\mathrm{P}}(B)$, let $v$ be an eigenvector of $B$ for $e$ with finite support, whence it must be of full finite support. And all the cycles it supports must be totally finitely supported. Specifically, for the witness cycles we must have $w_{B}\left(c^{k}\right)=e$ after Corollary 3.11, claim 2. Whence for any node in a witness cycle $i \in \overline{n_{B}^{e}}$ we have $B_{i i}^{+}=e$. $(2 \Rightarrow 3)$ After Proposition 3.16, claim 2, $\mathcal{V}_{e}(B)=\left\langle\left\{B_{. i}^{+} \otimes v_{i} \mid i \in \bar{n}_{B}^{e}\right\}\right\rangle_{\overline{\mathcal{K}}}$ with $B_{. i}^{+} \otimes v_{i} \in \mathcal{V}_{e}(B)$. As $\overline{\mathcal{K}}$ is a semifield, there exists $v_{i}^{-1}$ so that $B_{. i}^{+} \in \mathcal{V}_{e}(B)$. $(3 \Rightarrow 1)$ Since $B_{i i}^{+}=e, B_{i i}^{+}$is a finitely-supported eigenvector of $B$ for $e$.

Proposition 3.20. Let $A \in \mathcal{M}_{n}(\overline{\mathcal{D}})$ be an irreducible matrix over a completed selective radicable semifield. Then $\Lambda^{\mathrm{P}}(A)=\left\{\mu_{\oplus}(A)\right\}=\mathrm{P}^{\mathrm{P}}(A)$.

Proof. Note that $\mu_{\oplus}(A)=\top$ is proper by Proposition 3.18. When $\mu_{\oplus}(A) \prec \top$, call $B=\tilde{A}^{\mu_{\oplus}(A)}$, so that by Lemma $3.14 \mu_{\oplus}(A)$ is proper for $A$ if and only if $e$ is proper for $B$. But this is warranted by Proposition 3.19 since it is clear that for $c \in C_{B}^{+}, w_{B}(c) \preccurlyeq e$ with witness cycles of $B$ for $e$ those of $A$ for $\mu_{\oplus}(A)$. For uniqueness, since $\mu_{\oplus}(A)$ is an eigenvalue for a critical (witness) cycle $\mu_{A}(c)=\mu_{\oplus}(A)^{l(C)}$. Clearly, for any other proper eigenvalue $\rho \preccurlyeq \mu_{\oplus}(A)$ by definition. After Corollary $3.11 \mu_{A}(c) \preccurlyeq \rho^{l(c)}$, hence $\mu_{\oplus}(A)^{l(C)} \preccurlyeq \rho^{l(C)}$. By the compatibility of order and product, then $\mu_{\oplus}(A) \preccurlyeq \rho$ which entails $\mu_{\oplus}(A)=\rho$.

Hence, call principal eigenvalue of an irreducible matrix $\mu_{\oplus}(A)=\mu_{A}^{\oplus}\left(C_{A}^{+}\right)$the greatest of its cycle means. Since every eigenvector is fully supported, witness cycles must all be critical. In such case, we say that $\operatorname{FEV}_{\mu_{\oplus}(A)}(A)=$ $\mathbf{n}_{A}^{\mu_{\oplus}(A)}=\mathbf{n}_{\tilde{A}}^{e}=\left\{B_{i}^{+} \mid B_{i i}^{+}=e\right\}$ are the fundamental eigenvectors of $A$ for $\mu_{\oplus}(A) \prec \top$, since clearly $\mathcal{V}_{\mu_{\oplus}(A)}(A)=$ $\left\langle\operatorname{FEV}_{\mu_{\oplus}(A)}(A)\right\rangle_{\overline{\mathcal{K}}}$. By Corollary 3.20, if $e \in \mathrm{P}^{\mathrm{P}}(B)$ we may call the witness cycles of $B$ for $e$ critical and matrix $B$ itself is definite [29] or normalized [35].

\subsubsection{Discussion: bases vs. generators, eigenspace schematics and spectral lattices}

Usual spectral theory in incomplete idempotent semifield proceeds by looking for a basis among the generators of the eigenspace, as a mechanism to find a minimal representation for the eigenspaces [36,37]:

Proposition 3.21. Let $B \in \mathcal{M}_{n}(\overline{\mathcal{K}})$ be an irreducible matrix over a completed selective semifield such that $e \in \mathrm{P}^{\mathrm{P}}(B)$. Then:

1. If $i, j$ are vertices in witness cycles for $e$, then $B_{. i}^{+}=\alpha \otimes B_{. j}^{+}, \alpha \neq \top$ if and only if they belong to the same witness cycle $c \in C_{B}^{+}$.

2. The set of distinct fundamental eigenvectors of $B$ for e obtained from each witness cycle $c, \mathbf{n}_{B}^{e}(c)$, is a finite chain, that is, a totally-ordered set.

3. $\mathcal{V}_{e}(B)$ is generated by a subset of the fundamental eigenvectors obtained by picking a single eigenvector from each witness cycle, $\mathbf{m}_{B}^{e}$. 
Proof. Regarding claim 1 (from [29, Theorem 4.3.3]), let $i, j \in \bar{n}$ be nodes in witness cycles, that is $B_{i i}^{+}=e=B_{j j}^{+}$, and $B_{. i}^{+}=\alpha \otimes B_{. j}^{+}$. Then $B_{j i}^{+}=\alpha \otimes B_{j j}^{+}=\alpha$ and $B_{i i}^{+}=\alpha \otimes B_{i j}^{+}=e$, hence $B_{i j}^{+}=\alpha^{-1}$. Therefore, a path $c=(i \rightsquigarrow j)$, $(j \rightsquigarrow i)$ has maximum weight $w_{B^{+}}(c)=w_{B^{+}}\left(c_{i j}\right) \otimes w_{B^{+}}\left(c_{j i}\right)=\alpha \otimes \alpha^{-1}=e$ whence $c$ is a witness cycle and $i, j \in V_{c}$. On the other hand, if $i, j \in V_{c}, c \in C_{B}^{+}$with $w_{B^{+}}(c)=e$, call $c_{i j}$ that path in the cycle from $i$ to $j$, so that $B_{i j}^{+}=w_{B^{+}}\left(c_{i j}\right)=\alpha \prec \top$. By definition $B_{k i}^{+} \succcurlyeq B_{k j}^{+} \otimes \alpha$, but if $B_{k i}^{+} \succ B_{k j}^{+} \otimes \alpha$, since $\perp \prec \alpha \prec \top$, we would have $B_{k j}^{+} \prec B_{k i}^{+} \otimes \alpha^{-1}$, contradicting that $c_{i j}$ is maximal, so $B_{k i}^{+}=B_{k j}^{+} \otimes \alpha$. Since $k$ is generic, the claim follows.

For claim 2, fix $i \in V_{c}$ and $c$ a witness cycle, and notice from the previous paragraph that the scalar in $B_{\cdot i}^{+}=B_{\cdot j}^{+} \otimes \alpha$ is precisely $\alpha=w_{B^{+}}\left(c_{i j}\right)$. Given a matrix over an entire zerosumfree semiring $\mathcal{S}$, choose any $i$ in a cycle $c$ and define the set of path weights from $i$ as $W_{A}^{i}(c)=\left\{w_{A}\left(c_{i j}\right) \mid j \in V_{c}\right\}$. In the set of weights $W_{B^{+}}^{i}(c)=\left\{w_{B^{+}}\left(c_{i j}\right)\right\}_{j \in c}-$ including $w_{B^{+}}\left(c=c_{i i}\right)=e$-if we have $w_{B^{+}}\left(c_{i j}\right) \neq w_{B^{+}}\left(c_{i k}\right)$ for $j \neq k$ this implies $B_{\cdot j}^{+} \neq B_{\cdot k}^{+}$and vice versa. Hence $\mathbf{n}_{B}^{e}(c)=\left\{\alpha \otimes B_{. i}^{+} \mid \alpha \in W_{B^{+}}^{i}(c)\right\}$ has $\left|W_{B^{+}}(c)\right| \preccurlyeq n$ elements so, finally, order the fundamental eigenvectors by the proportionality constants $\alpha_{j}$ turning the set into an induced total order. If $\mathcal{S}$ is commutative and incomplete we can dispense with specifying the starting node for $W_{A}(c)$, since every choice of $i$ generates the same set of values.

Regarding claim 3, from claim 1 we know that all the fundamental eigenvectors provided by a witness cycle $c^{k}$ can be generated by a single eigenvector in that cycle $\mathbf{m}_{B}^{e}\left(c^{k}\right)$ since these are all proportional. Agreeing with claim 3 the set of these $\mathbf{m}_{B}^{e}=\bigcup_{c^{k} \in C_{B}^{c}} \mathbf{m}_{B}^{e}\left(c^{k}\right)$ is enough to generate the eigenspace, $\mathcal{V}_{e}(B)=\left\langle\mathbf{m}_{B}^{e}\right\rangle_{\overline{\mathcal{K}}}$

\section{By Lemma 3.14 and Proposition 3.21,}

Corollary 3.22. Let $A \in \mathcal{M}_{n}(\overline{\mathcal{K}})$ be an irreducible matrix over a completed selective semifield such that $\mu_{\oplus}(A) \prec \top$. Then $\mathbf{n}_{A}^{\mu_{\oplus}(A)} \supseteq \mathbf{m}_{A}^{\mu_{\oplus}(A)}$, and $\mathbf{m}_{A}^{\mu_{\oplus}(A)}=\mathbf{m}_{\tilde{A}}^{e}$ are a basis for $\mathcal{V}_{\mu_{\oplus}(A)}(A), \mathcal{V}_{\mu_{\oplus}(A)}(A)=\left\langle\mathbf{m}_{A}^{\mu_{\oplus}(A)}\right\rangle_{\overline{\mathcal{K}}}$.

However, in complete idempotent semifields the technique sketched in Proposition 3.21 cannot be used with $\mu_{\oplus}(A)=\top$, since when $w_{A}(c)=\top$ the set $W_{A}(c)$ may be reduced to $W_{A}(c)=\{\top\}$ when $i$ is such that the weight to the next $j$ in the cycle is $\top$. Furthermore, finding a basis for the top (proper) eigenvalue may be problematic as shown in Example 7.

Even if a basis is found, a continuous semimodule may be difficult to visualize, since, on the one hand, only techniques for low-dimensional spaces are known-like Mairesse's projection [38]—and, on the other, these mostly overlook the order properties of eigenvectors. For instance, for a vector $v \in \mathcal{S}^{n}$, call the ray of $v$ the set $\lambda v=\{\lambda \otimes v \mid$ $\lambda \in S\}$. Clearly vectors in the same ray are linearly dependent and the basis extraction process directly addresses this issue by keeping a single generating vector per ray [36]. However, in complete idempotent semimodules different rays with the same support meet at two points, $\{\perp \otimes v, \top \otimes v\}$, unlike rays in incomplete semimodules (see Fig. 2), and these have completely different interpretations (null eigenvector and saturated eigenvector).

To overcome these limitations we propose the use of (eigenspace) schematics which are modified order diagrams where rays are represented as continua (dashed lines) and the joins are suggested by the overall order structure. Examples of this representation can be found in Section 4.

When order properties are important we propose an alternative representation for an eigenspace: the subsemimodule generated from the fundamental eigenvectors by the action of the complete idempotent subsemifield $B$, its (right) eigenlattice or spectral lattice,

$$
\mathcal{L}_{\mu_{\oplus}(A)}(A)=\left\langle\operatorname{FEV}_{\mu_{\oplus}(A)}(A)\right\rangle_{\beta} .
$$

Furthermore, if we consider for improper $\rho \in \mathrm{P}(A) / \mathrm{P}^{\mathrm{P}}(A)$ and call the saturated eigenvectors $\mathrm{FEV}_{\rho}(A)=$ $\top \otimes \operatorname{FEV}_{\mu_{\oplus}(A)}(A)=\left\{T^{n}\right\}$, we can check that

$$
\mathcal{L}_{\rho}(A)=\left\langle\operatorname{FEV}_{\rho}(A)\right\rangle_{\beta}=\mathcal{V}^{\top}(A)
$$

For such finite lattices we have strong representation theorems in terms of the sets of join and meet irreducibles [1, Theorem 3, p. 20]. Taking these in consideration, our results can be summarized as,

Theorem 3.23. Let $A \in \mathcal{M}_{n}(\overline{\mathcal{K}})$ be an irreducible matrix over a complete selective radicable semifield, $\overline{\mathcal{K}}$. Then, 


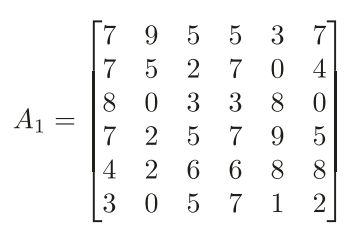

(a) An irreducible matrix

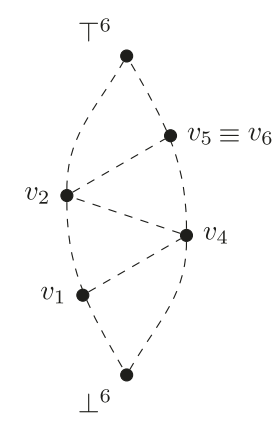

(c) Schematic of $\mathcal{V}_{\rho(A)}(A)$

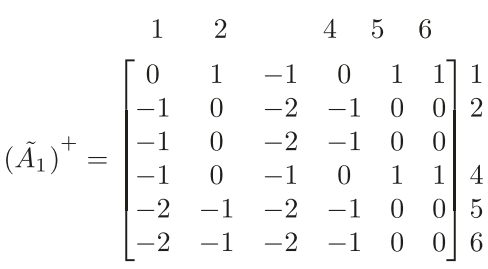

(b) Its normalized transitive closure

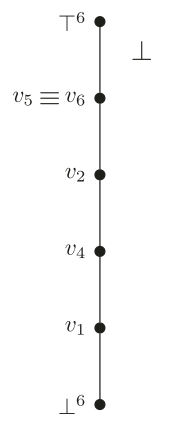

(d) $\mathcal{L}_{\rho(A)}(A) \cong 6$

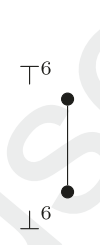

(e) $\mathcal{V}^{\top}(A)=\mathcal{L}^{\top}(A) \cong 2$

Fig. 2. Spectrum of an irreducible matrix with finite $\rho\left(A_{1}\right)=8$. The irreducible matrix (a), its normalized transitive closure (b) with the left and right eigenvectors indicated in the row and column numbers, a schematics of the right eigenspace (c) and its right eigenlattice (d). The lattice for an improper eigenvalue is reduced to 2 as shown in (e).

1. $\Lambda(A)=\overline{\mathcal{K}} \backslash\{\perp\}=\mathrm{P}(A)$.

2. $\Lambda^{\mathrm{P}}(A)=\left\{\mu_{\oplus}(A)\right\}=\mathrm{P}^{\mathrm{P}}(A)$.

3. If $\rho \in \mathrm{P}(A) \backslash \mathrm{P}^{\mathrm{P}}(A)$, then $\mathcal{V}_{\rho}(A)=\left\{\perp^{n}, \top^{n}\right\}=\mathcal{L}_{\rho}(A)$.

4. If $\mu_{\oplus}(A) \prec \top$, then $\mathcal{V}_{\rho(A)}(A)=\left\langle\operatorname{FEV}_{\rho(A)}(A)\right\rangle_{\overline{\mathcal{K}}} \supset \mathcal{L}_{\rho(A)}(A)=\left\langle\operatorname{FEV}_{\rho(A)}(A)\right\rangle_{B}$.

Proof. Claim 1 follows from Corollary 2.8 and Corollary 3.8. Claim 2 is Proposition 3.20. Claim 3 is claim 2 of Proposition 3.17 and claim 4 follows from Lemma 3.14, Corollary 3.22 and the paragraphs after it describing the spectral lattices.

\section{Examples}

We next provide some examples over the completed max-plus algebra $\bar{R}_{\max ,+}$, being, to the extent of our knowledge, the most widespread completed idempotent semifield, going also by the name of min-max-plus [39], minimax [27] or morphological algebra [6].

Example 6. (From [29, Example 4.3.7].) The matrix in $\overline{\mathbb{R}}_{\max ,+}$ in Fig. 2 is irreducible with $\rho(A)=8$.

The maximal cycle mean is $\rho(A)=\lambda(A)=8$ and the critical cycles are $C_{A}^{c}=\left\{c_{1}, c_{2}, c_{3}\right\}$ with $c_{1}=(1 \rightarrow 2 \rightarrow 1)$, $c_{2}=(4 \rightarrow 5 \rightarrow 6 \rightarrow 4), c_{3}=(5 \circlearrowright)$. Since the loop $c_{3}$ has its vertices included in those of $c_{2}$, only the latter is considered.

Call $v_{i}=\tilde{A}_{\cdot i}$. The first cycle generates the chain $v_{1}<v_{2}$ with $W_{\tilde{A}}^{1}\left(c_{1}\right)=\{0,1\}$ and the second cycle generates $v_{4}<v_{5}=v_{6}$ with $W_{\tilde{A}}^{4}\left(c_{2}\right)=\{0,1\}$, so that $v_{2}=1 \otimes v_{1}$ and $v_{5} \equiv v_{6}=1 \otimes v_{4}$. Furthermore $v_{5} \equiv v_{6}>v_{2}>v_{4}>v_{1}$ so a schematic representation of the eigenspace will look like Fig. 2c.

For proper $\rho(A)=8 \operatorname{FEV}_{\rho(A)}(A)=\bar{n}_{A}^{\rho(A)}=\{1,2,4,5\}$ so that if we select $\bar{m}_{A}^{\rho(A)}=\{1,4\}$ the (right) eigenspace is $\mathcal{V}_{\rho(A)}(A)=\left\langle v_{1}, v_{4}\right\rangle_{\overline{\mathbb{R}}_{\max ,+}}$ and the spectral lattice is $\mathcal{L}_{\rho(A)}(A) \cong\left\langle v_{1}, v_{2}, v_{4}, v_{5}\right\rangle_{\mathbb{B}} \cong \mathbb{G}$, whereas for an improper $\rho \in \mathrm{P}(A) \backslash \mathrm{P}^{\mathrm{P}}(A)$ the eigenspace and the spectral lattice are reduced to $\perp^{6}<\top^{6}$.

But the eigenspace for $\rho(A)=\top$ will not admit a compact expression in some cases: As Example 7 shows, the situation for a proper top eigenvalue is more complicated. 


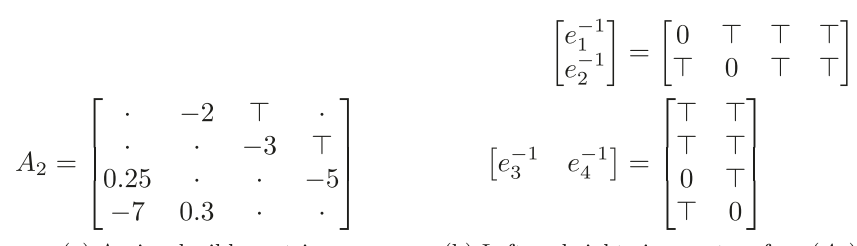

(a) An irreducible matrix

(b) Left and right eigenvectors for $\rho\left(A_{2}\right)=T$

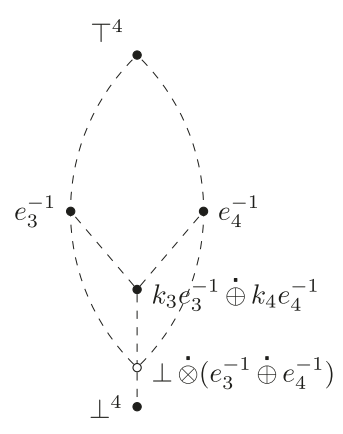

(c) Schematics of $\mathcal{V}_{\top}\left(A_{2}\right)$

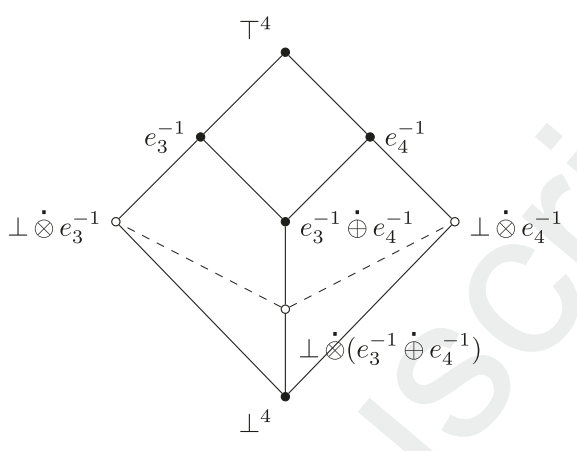

(d) $\mathcal{L}_{\mathrm{T}}\left(A_{2}\right)=\left\langle\left\{e_{3}^{-1}, e_{4}^{-1}\right\}\right\rangle_{\mathbb{R}_{\min ,+}} \cup\left\{\perp^{4}\right\}$

Fig. 3. An irreducible matrix with $\rho\left(A_{2}\right)=\top$, (a), some left and right eigenvalues (b), the schematics of the right eigenspace (c) and the representation of the eigenlattice (d). The saturated eigenspace $\mathcal{V}^{\top}\left(A_{2}\right)$ is not represented.

Example 7. Matrix $A_{2}$ with entries in $\overline{\mathbb{R}}_{\max ,+}$ in Fig. 3 is irreducible with $\rho\left(A_{2}\right)=\top$, therefore $A_{2}^{+}=\top^{4 \times 4}$. A top cover for columns is $\{3,4\}$ and a top cover for rows is $\{1,2\}$, so $\left\{e_{3}^{-1}, e_{4}^{-1}\right\} \in \mathcal{V}_{\top}\left(A_{2}\right)$. But note that for finite $k_{3}, k_{4} \in \overline{\mathbb{R}}_{\max ,+}, K_{3} \otimes e_{3}^{-1} \dot{\oplus} K_{4} \otimes e_{4}^{-1} \in \mathcal{V}_{\top}\left(A_{2}\right)$ although it cannot be obtained as an $\overline{\mathbb{R}}_{\max ,+}$-linear combination from $\left\{e_{3}^{-1}, e_{4}^{-1}\right\}$.

Note that for matrix $A_{2}$ in Fig. $3, \top \dot{\otimes} e_{3}^{-1}$ does not belong to $\mathcal{V}_{\top}\left(A_{2}\right)$ but for any other $k_{3} \in \mathbb{R}_{\min ,+}, k_{3} \dot{\otimes} e_{3}^{-1}$ does, and similarly with $k_{4} \in \mathbb{R}_{\min ,+}$, as well as their min-plus combinations. This seems to indicate that $\mathcal{V}_{\top}\left(A_{2}\right)$ behaves like a ( $n$ incomplete) min-plus space, to which a $\perp^{4}$ has been added,

$$
\mathcal{V}_{\top}\left(A_{2}\right)=\left\langle\left\{e_{3}^{-1}, e_{4}^{-1}\right\}\right\rangle_{\mathbb{R}_{\min ,+}} \cup\left\{\perp^{4}\right\}=\left\langle\left\{\perp^{4}, e_{3}^{-1}, e_{4}^{-1}\right\}\right\rangle_{\mathbb{R}_{\min ,+}} .
$$

Of course, this is the inverse of a ( $n$ incomplete) max-plus space completed with a top, $\mathcal{V}_{\top}\left(A_{2}\right)=$ $\left\langle\left\{e_{3}, e_{4}\right\}\right\rangle_{\mathbb{R}_{\max ,+}} \cup\left\{T^{4}\right\}^{-1}$.

\section{Discussion: the spectral problem in incomplete idempotent semifields}

Recall from Section 2.1 that an idempotent semifield $\mathcal{K}$ is an idempotent semiring whose multiplicative structure is a commutative group, and that these are all incomplete, unless completed (cf. Section 2.2). The characterization of the spectra of matrices over incomplete idempotent semifields has been developed, among others, by [13,26,27,34,40-44] for the $\mathbb{R}_{\max , \times}, \mathbb{R}_{\max ,+}$ or $\mathbb{R}_{\min ,+}$ cases. Ref. [45] related the $\mathbb{R}_{\max ,+}$ and $\mathbb{R}_{\max , \times}$ cases with the Perron-Frobenius spectral theory. Most of this work has been done for the irreducible matrix case, although some works address the reducible case $[34,42,45,46]$ — but $[14,29]$ are the most up-to-date presentations. It remains at the basis of a number of applications in optimization [40], discrete event systems [41], classification [14], and data mining [7]. Only Ref. [10] addresses the issue of concept lattices.

As a concrete instance of commutative idempotent semifields, we choose $\mathbb{R}_{\max ,+}$ whose spectral theory already shows notorious differences with the spectral theory over commutative fields, since the eigenvalue equations become:

$$
\max _{1 \leq j \leq n}\left\{A_{i j}+v_{j}\right\}=v_{i}+\rho, \quad \forall 1 \leq i \leq n \quad \max _{1 \leq i \leq n}\left\{u_{i}+A_{i j}\right\}=\lambda+u_{j}, \quad \forall 1 \leq j \leq n .
$$


The spectral description of irreducible matrices is the building block for other spectra. Irreducible matrices are those not permutationally equivalent to block triangular matrices (see Lemma 2.7). A well-known result in incomplete idempotent semifields is:

Proposition 5.1 (Spectrum of irreducible matrices over incomplete idempotent semifields). Let $A \in \mathcal{K}^{n \times n}$ be an irreducible matrix with entries in a commutative, selective, radicable idempotent semifield. Then,

1. $\Lambda(A)=\left\{\mu_{\oplus}(A)\right\}=\mathrm{P}(A)$.

2. $\mathcal{V}_{\mu_{\oplus}(A)}(A)=\left\langle\operatorname{FEV}_{\mu_{\oplus}(A)}(A)\right\rangle_{\mathcal{K}}$.

Proof sketch. Call $\mu_{\oplus}(A)$ the extremal cycle mean of the matrix considered as a graph, which is guaranteed to exist and be finite if we further demand that $\mathcal{K}$ be radicable (Section 2.4.3), that is, semirings in which equation $a^{b}=c$ can be solved for $a$.

Since $\mathcal{K}$ is a semiring we may define a normalized $\tilde{A}=A / \mu_{\oplus}(A)$ guaranteed to have a transitive closure $\tilde{A}^{+}$and an efficient way to find it (Section 2.4.2). Now, the columns of $\tilde{A}^{+}$with a diagonal unit entry are called the fundamental eigenvectors of $A$ for $\mu_{\oplus}(A), \operatorname{FEV}_{\mu(A)}(A)$, which can be proven to generate the eigenspace (Section 3.2.1). The actual proof can be consulted in $[14, \stackrel{\oplus}{ }$ Chap. $6, \S 4]$.

Proposition 5.1 is in stark contrast with our main result, Theorem 3.23, and the differences seem to be deeper when we consider the case of reducible matrices, a matter for future consideration.

\section{Conclusions}

The spectra of matrices over complete commutative selective radicable semifields show already notable differences with respect to that over incomplete semifields. First there is the topic of improper eigenvalues and the proper/improper distinction; then the question of saturated supports giving richness to eigenspaces; and finally the issue of the structure added to eigenspaces due to the order relation between vectors: these are already complete continuous lattices.

This suggests introducing a complementary approach to that of describing eigenspaces by their bases by considering certain complete subsemimodules, viz. the eigenlattices or spectral lattices.

\section{Acknowledgements}

We would like to thank the reviewers of the paper having contributed to its clarity and quality.

FJVA was partially supported by EU FP7 project LiMoSINe (contract 288024) for this research. CPM was partially supported by the Spanish Government-Comisión Interministerial de Ciencia y Tecnología project 2011-268007/TEC.

\section{References}

[1] B. Ganter, R. Wille, Formal Concept Analysis: Mathematical Foundations, Springer, Berlin, Heidelberg, 1999.

[2] M. Barbut, B. Monjardet, Ordre et classification. Algèbre et combinatoire, tome I, Méthodes mathématiques des sciences de l'Homme, Hachette, 1970

[3] J. Poelmans, S.O. Kuznetsov, D.I. Ignatov, G. Dedene, Formal concept analysis in knowledge processing: a survey on models and techniques, Expert Syst. Appl. 40 (16) (2013) 6601-6623.

[4] A. Burusco, R. Fuentes-González, The study of the L-fuzzy concept lattice, Mathw. Soft Comput. 1 ( 3 ) (1994) $209-218$.

[5] R. Bolohlávek, Fuzzy Relational Systems. Foundations and Principles, IFSR International Series on Systems Science and Engineering, vol. 20, Kluwer Academic, 2002.

[6] J.S. Golan, Semirings and Their Applications, Kluwer Academic, 1999.

[7] F.J. Valverde-Albacete, C. Pelaez-Moreno, Extending conceptualisation modes for generalised formal concept analysis, Inf. Sci. 181 (2011) 1888-1909.

[8] J.A. Goguen, L-fuzzy sets, J. Math. Anal. Appl. 18 (1) (1967) 145-174.

[9] M. Gondran, M. Minoux, Dioids and semirings: links to fuzzy set and other applications, Fuzzy Sets Syst. 158 (2007) $1273-1294$.

[10] F.J. Valverde-Albacete, C. Pelaez-Moreno, Spectral lattices of $\left(R_{\max },+\right)$-formal contexts, in: Formal Concept Analysis. Proceedings of the 6th International Conference, ICFCA 2008, Montreal, Canada, February 25-28, 2008, in: Lecture Notes in Computer Science (LCNS), vol. 4933, 2008, pp. 124-139. 
[11] R. Belohlavek, V. Vychodil, Discovery of optimal factors in binary data via a novel method of matrix decomposition, J. Comput. Syst. Sci. 76 (1) (2010) 3-20.

[12] R. Belohlavek, Optimal decompositions of matrices with entries from residuated lattices, J. Log. Comput. 22 (6) (2012) $1405-1425$.

[13] M. Gondran, M. Minoux, Valeurs propres et vecteurs propres dans les dioïdes et leur interprétation en théorie des graphes, EDF, Bull. Dir. Etudes Rech., Ser. C, Math. Inform. 2 (1977) 25-41.

[14] M. Gondran, M. Minoux, Graphs, Dioids and Semirings. New Models and Algorithms, Operations Research Computer Science Interfaces Series, Springer, 2008.

[15] F.J. Valverde-Albacete, C. Peláez-Moreno, Spectral lattices of reducible matrices over completed idempotent semifields, in: M. Ojeda-Aciego Outrata (Eds.), Concept Lattices and Applications, CLA 2013, 2013, pp. 211-224, http://ceur-ws.org/Vol-1062/paper18.pdf.

[16] J.S. Golan, Semirings and Affine Equations over Them: Theory and Applications, Mathematics and Its Applications, Kluwer Academic Publishers, 2003.

[17] J.S. Golan, Power Algebras over Semirings. With Applications in Mathematics and Computer Science, Mathematics and Its Applications, vol. 488, Kluwer Academic, Dordrecht, Boston, London, 1999.

[18] R.A. Cuninghame-Green, K. Cechlárová, Residuation in fuzzy algebra and some applications, Fuzzy Sets Syst. 71 (2) (1995) $227-239$.

[19] R.A. Cuninghame-Green, Minimax algebra and applications, Fuzzy Sets Syst. 41 (3) (1991) 251-257.

[20] U. Hebisch, H. Weinert, Semirings. Algebraic Theory and Applications in Computer Science, Series in Algebra, vol. 5, World Scientific, 1998.

[21] W. Kuich, A. Salomaa, Semirings, Automata, Languages, EATCS Monographs on Theoretical Computer Science, Springer-Verlag, hg Berlin, Heidelberg, New York and Tokyo, 1986.

[22] J.J. Moreau, Inf-convolution, Sous-additivité, convexité des fonctions Numériques, J. Math. Pures Appl. 49 (1970) $109-154$.

[23] I. Singer, Abstract Convex Analysis, Monographs and Advanced Texts, Wiley-Interscience, 1997.

[24] I. Singer, (*s)-Dualities, J. Math. Sci. 115 (4) (2003) 2506-2541.

[25] I. Singer, Some relations between linear mappings and conjugations in idempotent analysis, J. Math. Sci. 115 (5) (2003) 2610-2630.

[26] N. Vorobjev, The extremal matrix algebra, Dokl. Akad. Nauk SSSR 4 (1963) 1220-1223.

[27] R. Cuninghame-Green, Minimax Algebra, Lecture Notes in Economics and Mathematical Systems, vol. 166, Springer, 1979.

[28] G. Cohen, S. Gaubert, J.-P. Quadrat, Duality and separation theorems in idempotent semimodules, Linear Algebra Appl. 379 (2004) $395-422$.

[29] P. Butkovic, Max-linear Systems. Theory and Algorithms, Monographs in Mathematics, Springer, 2010.

[30] J.-S. Duan, The transitive closure, convergence of powers and adjoint of generalized fuzzy matrices, Fuzzy Sets Syst. 145 (2) (2004) 301-311.

[31] R.A. Brualdi, Combinatorial matrix theory, Chap. 27, in: [47], 27.1-27.12, 2007.

[32] J.L. Stuart, Digraphs and matrices, Chap. 29, in: [47], 29-1-29-14, 2007.

[33] B. Davey, H. Priestley, Introduction to Lattices and Order, 2nd edn., Cambridge University Press, Cambridge, UK, 2002.

[34] R.B. Bapat, T.E.S. Raghavan, Nonnegative Matrices and Applications, Encyclopedia of Mathematics and Its Applications, vol. 64, Cambridge University Press, 1997.

[35] M. Akian, R. Bapat, S. Gaubert, Max-plus algebra, Chap. 25, in: [47], 25-1-25-17, 2007.

[36] R.A. Cuninghame-Green, P. Butkovi c, Bases in max-algebra, Linear Algebra Appl. 389 (2004) 107-120.

[37] S. Sergeev, Max-plus definite matrix closures and their eigenspaces, Linear Algebra Appl. 421 (2-3) (2007) 182-201.

[38] J. Mairesse, A graphical approach of the spectral theory in the (max, +) algebra, IEEE Trans. Autom. Control 40 (10) (1995) $1783-1789$.

[39] B. Heidergott, G.J. Olsder, J.W. van der Woude, Max plus at work, modeling and analysis of synchronized systems: a course on maxplus algebra and its applications, Princeton Univ. Pr., 2006.

[40] U. Zimmermann, Linear and Combinatorial Optimization in Ordered Algebraic Structures, North Holland, 1981.

[41] F. Baccelli, G. Cohen, G. Olsder, J. Quadrat, Synchronization and Linearity, Wiley, 1992.

[42] S. Gaubert, Théorie des systèmes linéaires dans les dioïdes, Thèse, Thèse, École des Mines de Paris, 1992.

[43] G. Olsder, K. Roos, R.-J. van Egmond, An efficient algorithm for critical circuits and finite eigenvectors in the max-plus algebra, Linear Algebra Appl. 295 (1-3) (1999) 231-240.

[44] N.K. Krivulin, Eigenvalues and eigenvectors of matrices in idempotent algebra, Vestn. St. Petersbg. Univ., Math. 39 (2) (2006) 72.

[45] R. Bapat, A max version of the Perron-Frobenius theorem, Linear Algebra Appl. 275-276 (1998) 3-18.

[46] P. Butkovic, R.A. Cuninghame-Green, S. Gaubert, Reducible spectral theory with applications to the robustness of matrices in max algebra, SIAM J. Matrix Anal. Appl. 31 (3) (2009) 1412-1431.

[47] L. Hogben (Ed.), Handbook of Linear Algebra, Discrete Mathematics and Its Applications, Chapman \& Hall/CRC, 2007. 